University of Tennessee Health Science Center

UTHSC Digital Commons

\title{
Assessing the Sensitivity of the Canadian Adverse Event Following Immunization Surveillance System ( CAEFISS)
}

\author{
Mina Tadrous \\ University of Tennessee Health Science Center
}

Follow this and additional works at: https://dc.uthsc.edu/dissertations

Part of the Health Services Research Commons, and the International Public Health Commons

\section{Recommended Citation}

Tadrous, Mina , "Assessing the Sensitivity of the Canadian Adverse Event Following Immunization Surveillance System ( CAEFISS)" (2010). Theses and Dissertations (ETD). Paper 262. http://dx.doi.org/ 10.21007/etd.cghs.2010.0309.

This Thesis is brought to you for free and open access by the College of Graduate Health Sciences at UTHSC Digital Commons. It has been accepted for inclusion in Theses and Dissertations (ETD) by an authorized administrator of UTHSC Digital Commons. For more information, please contact jwelch30@uthsc.edu. 


\title{
Assessing the Sensitivity of the Canadian Adverse Event Following Immunization Surveillance System ( CAEFISS)
}

\begin{abstract}
Background: Vaccines are important to public health, but because of the way they are manufactured, their mechanism of action, and their indicated population, careful monitoring of their adverse events is necessary. Canada has a national surveillance system that collects reports on adverse events that may be associated with vaccine administration. Sensitivity is one of the tools used with surveillance systems to study the extent and characteristics of reporting of a surveillance system. To date, the sensitivity of the Canadian system has not been assessed.

Purpose: To assess the sensitivity of the Canadian Adverse Event Following Immunization Surveillance System (CAEFISS).

Methods: Based on specific adverse events following immunization (AEFI) and vaccines chosen for the study, a thorough literature search was completed to find the best source which identifies expected rates of AEFI. Studies used were assessed based on quality and sample size. The expected rates of AEFI, in combination with public health estimates of vaccine coverage rates, were used to estimate the expected number of reports. The reports provided the actual number of events used to calculate the sensitivity. Sensitivity was compared based on year of administration, age group, and type of AEFI.
\end{abstract}

Results: The overall sensitivity of the CAEFISS varied from $1.0 \%$ to $136.6 \%$ for various AEFI for the years 1997 to 2008 . For influenza the sensitivity was found to be $93.6 \%$ and $136.3 \%$ for GBS and anaphylaxis respectively. For DTaP, the rates were found to be $15.0 \%, 1.0 \%$, and $21.2 \%$ for anaphylaxis, $\mathrm{HHE}$, and seizures respectively, and for MMR the rates were $16.5 \%, 52.7 \%$, and $12.7 \%$ in relation to anaphylaxis, thrombocytopenia, and seizures respectively.

Conclusions: This is the first assessment of the sensitivity of the CAEFISS, and this study found that the system has reasonable ability to detect AEFI on a national level. CAEFISS had comparable senstivity to other vaccine reporting systems. Many of the AEFI had sensitivity values higher than the $5 \%-10 \%$ range traditionally seen in other passive surveillance systems related to adverse events. The greatest variation of sensitivity was seen between vaccines. Rarity and timing of the AEFI may also impact the sensitivity. Variation of sensitivity and the variation found in the sensitivity analysis lend to the further development and implementations of case definitions for rarer adverse events, especially anaphylaxis. Further research of other factors that impact reporting is necessary.

\section{Document Type}

Thesis

\section{Degree Name}

Master of Science (MS)

\section{Program}

Health Outcomes and Policy Research

\section{Research Advisor}

Junling Wang, Ph.D.

\section{Keywords}

Adverse Events, Canada, Public Health, Sensitivity, Surveillance System, Vaccine Safety 


\section{Subject Categories}

Health Services Research | International Public Health | Medicine and Health Sciences | Public Health 
ASSESSING THE SENSITIVITY OF THE CANADIAN ADVERSE EVENT

FOLLOWING IMMUNIZATION SURVEILLANCE SYSTEM (CAEFISS)

\author{
A Thesis \\ Presented for \\ The Graduate Studies Council \\ The University of Tennessee \\ Health Science Center
}

\author{
In Partial Fulfillment \\ Of the Requirements for the Degree \\ Master of Science \\ From The University of Tennessee
}

By

Mina Tadrous

May 2010 


\section{ACKNOWLEDGMENTS}

Many thanks to my advisor, Dr. Wang. She was extremely helpful throughout this process and guided me well. She has always responded quickly too all of my questions, at all hours. I would also like to thank my committee members, Dr. Arnold and Dr. Hoffman. They were more than flexible to schedule the times to propose and defend my thesis and provided great input as to the direction of my thesis. I would also like to show my deepest gratitude to Dr. Law, who supported me whole-heartedly although she met with an unexpected workload. I am so grateful for the opportunity she has given me for a wonderful thesis.

Also, I would like to express my sincerest gratitude to all of those involved in my residency and masters program led by the University of Tennessee in conjunction with MedCommunications Inc., and St. Jude Children's Hospital. Without this program, I never would have had the opportunity to complete my degree. Special thanks to Dr. Beth Miller, Dr. Anne Hurley, and Dr. James Hoffman who were all essential to the success of my two years. Lastly, I would like to thank Dr. Katie Suda for her leadership and guidance in so many aspects of my two years. She helped me to keep calm through it all. Finally, to all of those who positively impacted me in any way, thank you. 


\begin{abstract}
Background: Vaccines are important to public health, but because of the way they are manufactured, their mechanism of action, and their indicated population, careful monitoring of their adverse events is necessary. Canada has a national surveillance system that collects reports on adverse events that may be associated with vaccine administration. Sensitivity is one of the tools used with surveillance systems to study the extent and characteristics of reporting of a surveillance system. To date, the sensitivity of the Canadian system has not been assessed.

Purpose: To assess the sensitivity of the Canadian Adverse Event Following Immunization Surveillance System (CAEFISS).

Methods: Based on specific adverse events following immunization (AEFI) and vaccines chosen for the study, a thorough literature search was completed to find the best source which identifies expected rates of AEFI. Studies used were assessed based on quality and sample size. The expected rates of AEFI, in combination with public health estimates of vaccine coverage rates, were used to estimate the expected number of reports. The reports provided the actual number of events used to calculate the sensitivity. Sensitivity was compared based on year of administration, age group, and type of AEFI.
\end{abstract}

Results: The overall sensitivity of the CAEFISS varied from $1.0 \%$ to $136.6 \%$ for various AEFI for the years 1997 to 2008 . For influenza the sensitivity was found to be $93.6 \%$ and $136.3 \%$ for GBS and anaphylaxis respectively. For DTaP, the rates were found to be $15.0 \%, 1.0 \%$, and $21.2 \%$ for anaphylaxis, HHE, and seizures respectively, and for MMR the rates were $16.5 \%, 52.7 \%$, and $12.7 \%$ in relation to anaphylaxis, thrombocytopenia, and seizures respectively.

Conclusions: This is the first assessment of the sensitivity of the CAEFISS, and this study found that the system has reasonable ability to detect AEFI on a national level. CAEFISS had comparable senstivity to other vaccine reporting systems. Many of the AEFI had sensitivity values higher than the 5\%-10\% range traditionally seen in other passive surveillance systems related to adverse events. The greatest variation of sensitivity was seen between vaccines. Rarity and timing of the AEFI may also impact the sensitivity. Variation of sensitivity and the variation found in the sensitivity analysis lend to the further development and implementations of case definitions for rarer adverse events, especially anaphylaxis. Further research of other factors that impact reporting is necessary. 


\section{TABLE OF CONTENTS}

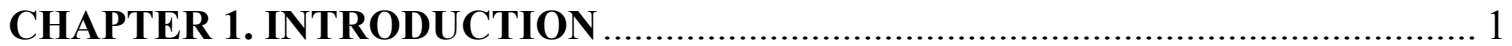

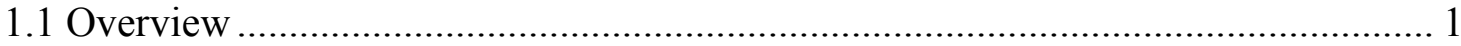

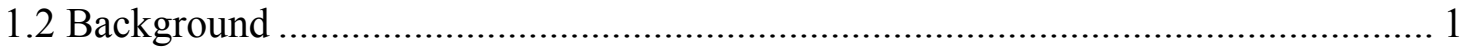

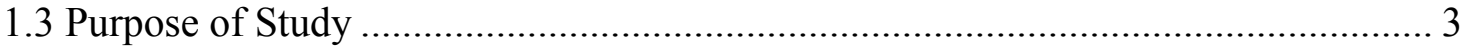

1.4 Specific Aims and Hypotheses.......................................................................... 3

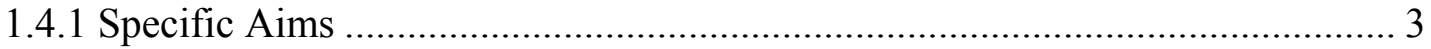

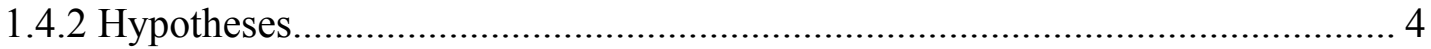

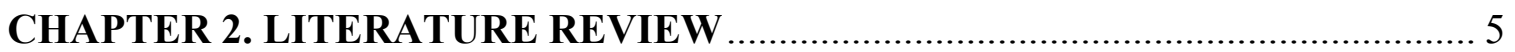

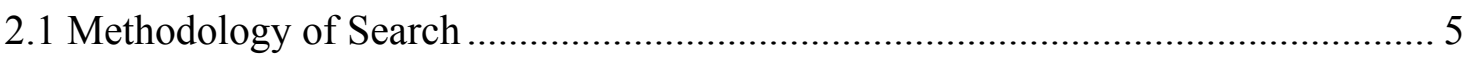

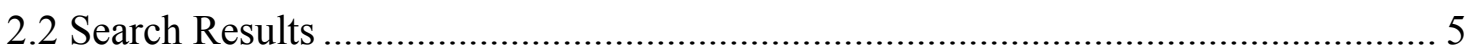

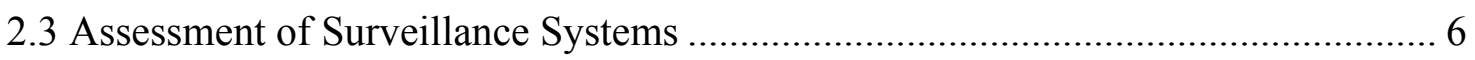

2.4 Assessing Sensitivity of Surveillance System...................................................... 7

2.5 Assessment of Drug Adverse Event Reporting Systems ........................................ 7

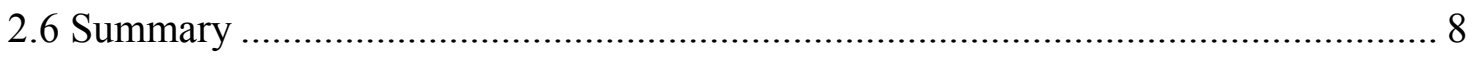

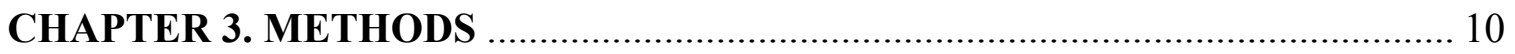

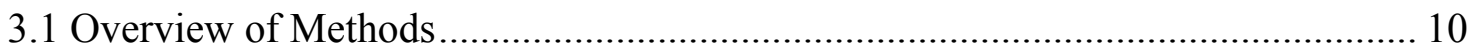

3.2 Estimating Expected Rates of AEFI............................................................... 12

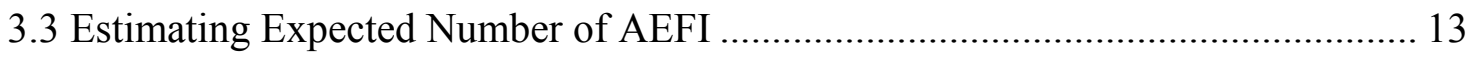

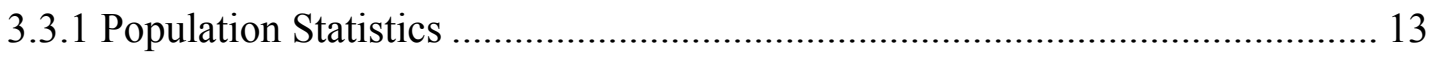

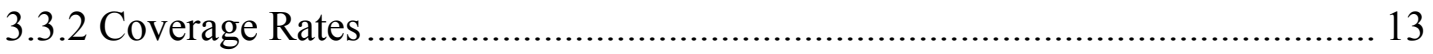

3.3.3 Estimating Doses Administered and Expected Number of Events .................. 14

3.4 Number of Events and Calculating Sensitivity ..................................................... 15

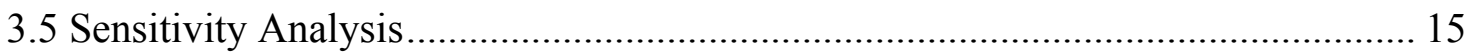

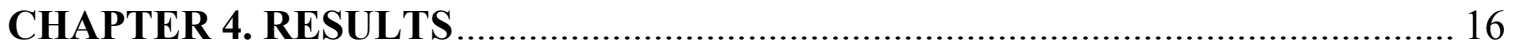

4.1 Expected Rates of AEFI......................................................................... 16

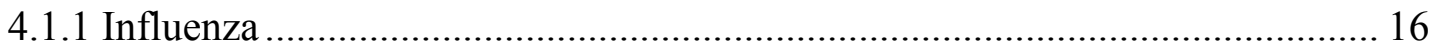

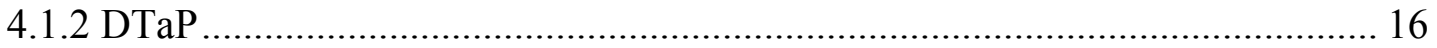

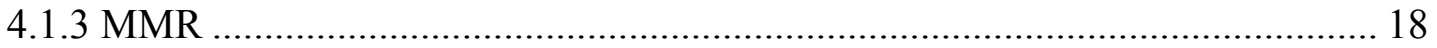

4.2 Estimated Number of Vaccines Administered ................................................. 18

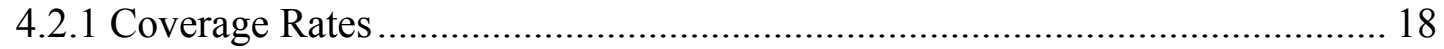


4.2.2 Targeted Population and Estimated Doses Administered ............................. 18

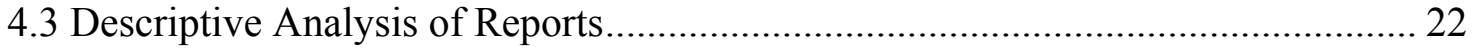

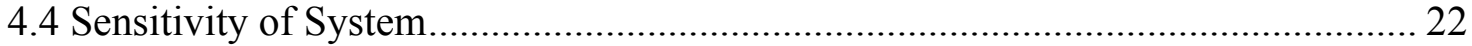

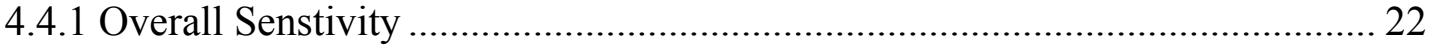

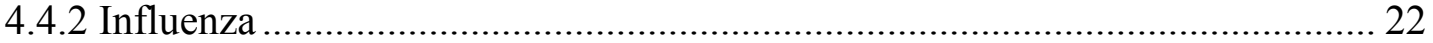

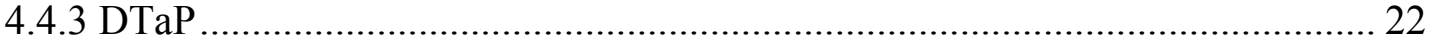

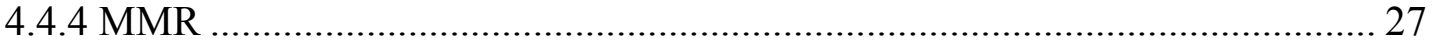

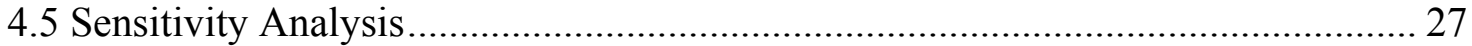

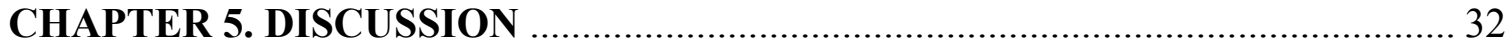

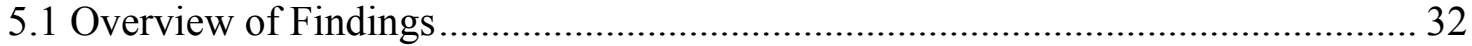

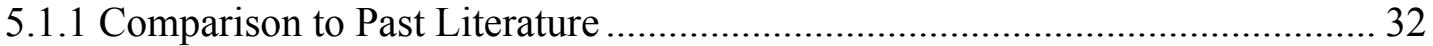

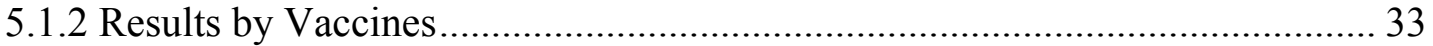

5.1.3 Adult versus Pediatric Reporting ................................................................ 35

5.1.4 Common versus Rare Adverse Events .......................................................... 35

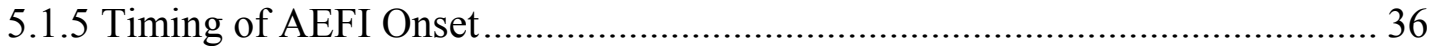

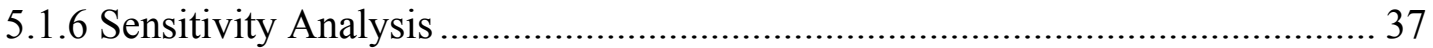

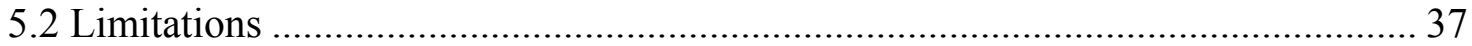

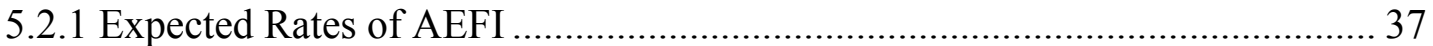

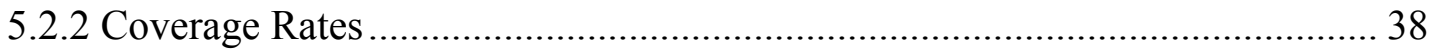

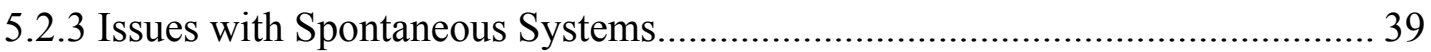

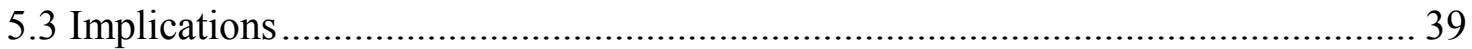

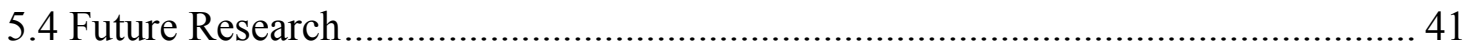

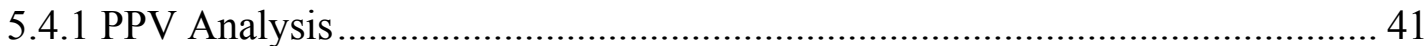

5.4.2 Policy and Reporter Assessment ............................................................... 41

5.4.3 Trending Analysis Using Moving Averages .............................................. 42

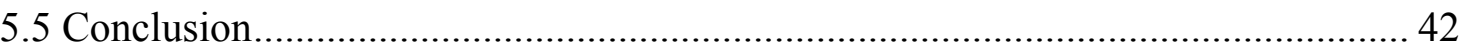

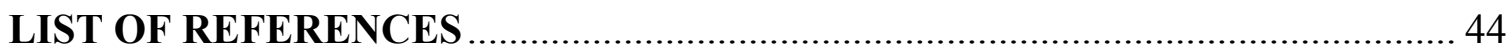

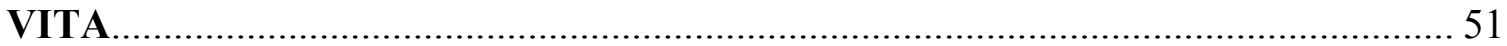




\section{LIST OF TABLES}

Table $3.1 \quad$ Vaccines and AEFI to be Analyzed by Temporal Association of AEFI

Table 4.1 Expected Rates of AEFI Based on Literature ........................................17

Table 4.2 Coverage Rates by Source and Year .................................................19

Table 4.3 Indicated Population and Total Doses per Year from 1997-2008 ...........21

Table 4.4 Descriptive Statistics of Selected Reports from 1997-2008 ....................23

Table 4.5 Number of Reports Received by CAEFISS between 1997-2008

Related to Selected Adverse Events by AEFI

Table 4.6 Number of Reports Received by CAEFISS between 1997-2008

Related to Selected Adverse Events by Reporters' Affiliation

Table 4.7 Total and Yearly Sensitivity by Vaccine and AEFI for 1997-2008

Table 4.8 One- and Two-way Sensitivity Analysis .30

Table 5.1 Ordered Expected Occurrence per Million Doses from Lowest to Highest .36

Table 5.2 Comparison of Estimated Influenza Vaccine Doses Administered to Reported Doses Distributed .38 


\section{LIST OF FIGURES}

Figure 3.1 Step-wise Approach to Analysis........................................................ 11

Figure 4.1 Estimated Canadian National Immunization Coverage

Rates for Influenza, DTaP, and MMR from 1997-2008 .......................20

Figure 4.2 Sensitivity for Anaphylaxis and GBS Related to Influenza

Immunizations

Figure 4.3 Sensitivity for Anaphylaxis, HHE, and Seizures Related to DTaP

Immunizations .28

Figure 4.4 Sensitivity for Anaphylaxis, Thrombocytopenia, and Seizures

Related to MMR Immunizations .28

Figure 5.1 Application of Moving Averages to GBS Related to Influenza Vaccination; 1-, 3-, and 5- Year Moving Averages.... 


\section{LIST OF ABBREVIATIONS}

$\mathrm{AE}$ Adverse Event

AEFI Adverse Event Following Immunization CAEFISS CCDR CCHS Canadian Adverse Event Following Immunization Surveillance System
$\ldots \ldots \ldots+. .1$ Canadian Communicable Disease Report $\mathrm{CDC}$ Canadian Adverse Event Following Immunization Surveillance System
$\ldots \ldots \ldots+. .1$ Canadian Communicable Disease Report

$\mathrm{DTaP}$ FDA GBS Canadian Adverse Event Following Immunization Surveillance System
$\ldots \ldots \ldots+. .1$ Canadian Communicable Disease Report

HHE Canadian Adverse Event Following Immunization Surveillance System
$\ldots \ldots \ldots+. .1$ Canadian Communicable Disease Report

HMO Hypotonic-Hyporesponsive Episode IMPACT $\mathrm{MeSH}$ MMR MMWR OP Food and Drug Administration ....Guillan-Barre Syndrome PHAC Health Maintenance Organization VAERS WHO Immunization Monitoring Program-Active Medical Subject Heading Measles, Mumps, and Rubella Vaccine CDC Morbidity and Mortality Weekly Report Oral Polio Vaccine Public Health Agency of Canada Vaccine Adverse Event Reporting System (U.S.) .. World Health Organization 


\section{CHAPTER 1. INTRODUCTION}

\subsection{Overview}

Vaccines are vital to public health, but because of their method of manufacturing, mechanism of action, and indicated population careful monitoring of their safety is important. Canada has a national surveillance system which collects reports on adverse events temporally associated with vaccine administration. An assessment of the sensitivity of the system has not been conducted to date. Such an assessment would allow for a better understanding of the system, identify areas for improvement, and reassure the public of the merit of such reporting systems. This thesis assesses the overall and annual sensitivity of the system for specific Adverse Event Following Immunization (AEFI).

\subsection{Background}

Vaccines are unique medical interventions. They are used for primary prevention of disease and are administered to healthy (non-diseased) individuals (Ellenberg \& Braun 2002). This is in contrast to most medical interventions which correct or treat a condition of illness. Vaccines are used worldwide, such that every person is recommended to receive multiple immunizations over his lifetime. The success of vaccines in reducing morbidity and mortality, globally, cannot be overstated. Their effects on overall life expectancy have far surpassed those of other medical interventions, especially within the pediatric population. Vaccines are biologic products with complex mechanisms of action and manufacturing processes (Grabenstein \& Grabenstein 1997; Jacobson 2003; Martin ,Nelson, Hershey, \& Engler 2003). Thus, although vaccines are generally safe, like any medical intervention, vaccines do present risk (Bonhoeffer \& Heininger 2007).

Public perception of vaccines is important. For vaccine effectiveness, herd immunity, defined as immunity in a large proportion of a population to ensure the continued suppression of disease outbreaks, must be maintained to. Therefore, any perception of vaccine risk or ineffectiveness may profoundly affect vaccine uptake, effectiveness, and public health. In some instances, vaccine use has been so effective that it may appear to the public that the disease has disappeared. This perception of disease eradication by vaccines has begun to undermine the value of immunization to the public (Fowler et al. 2008; Kimmel, Burns, \& Zimmerman 2003; O'Hagan \& Rappuoli 2004).

Vaccines are designed to induce an immune response, which is the basis for both harm and protection. Rare but serious adverse events can occur due to vaccines, but even large clinical studies that serve as the basis for approval may be underpowered to detect these events. Vaccine safety can be greatly affected by storage requirements and differences between lots, which require unique lot-by-lot monitoring (Letourneau, Wells, Walop, \& Duclos 2008; Mansoor 1999). Therefore, because of the importance of vaccines to public health, unique considerations, and challenges in identifying rare adverse events, postmarketing surveillance for vaccines is essential for public health and safety. 
Surveillance systems are one of the primary methods for post-marketing monitoring. Current AEFI surveillance systems rely on spontaneous reporting of AEFI, and therefore, these systems are not intended for the detection of new AEFI. Instead, passive surveillance systems focus on ongoing safety monitoring of the products. These systems generate associations between vaccines and adverse events and they cannot prove a cause and effect relationship. The value and appropriate application of spontaneous vaccine surveillance systems was exhibited with the Rotashield rotavirus vaccine. A surveillance system generated concern that Rotashield rotavirus vaccine increased the occurrence of intussusceptions. However, this increased risk was not confirmed until post-marketing studies were completed (Niu, Erwin, \& Braun 2001; Verstraeten et al. 2001; Zanardi, Haber, Mootrey, Niu, \& Wharton 2001). This approach is in contrast to other public health surveillance systems in which active surveillance is conducted in order to assess of the rate of occurrence of specific events (i.e. active surveillance (German 2000)).

Surveillance systems have been an integral tool in assuring vaccine safety, since they are designed for ongoing collection, analysis, interpretation, and application of data ("Immunization information systems progress--United States, 2006," 2008). These systems are capable of analyzing long-term trends and changes, but more importantly, are used for immediate action if a signal of increased risk becomes apparent. Although many specific attributes of reporting and public health systems have been evaluated, the analysis of sensitivity of an AEFI reporting system holds importance (German et al. 2001). To establish quality and dependability, the United States Center for Disease Control (CDC) published guidelines for the analysis of public health reporting and surveillance systems (German et al. 2001). While these guidelines focus on many aspects of public health surveillance systems, recommendations for sensitivity analysis are relevant to AEFI reporting.

CAEFISS was created to serve as a national monitoring system for reporting AEFIs. The current system allows healthcare professionals, consumers, public health workers, and vaccine manufacturers to report AEFIs. The CAEFISS objectives are to ensure continued safety of vaccines in Canada, monitor AEFI, identify any unusual rates of AEFI within specific vaccines and lots, provide timely information to healthcare providers to help weigh the risks and benefits of immunizations, and to identify specific areas that may require further epidemiological evaluations. Currently, the majority of CAEFISS is spontaneously reported, but a portion of the system is active through the IMPACT program. IMPACT is an active surveillance program which includes 12 Canadian hospitals accounting for $90 \%$ of all tertiary care pediatric beds in Canada. The implementation of the IMPACT program has increased awareness of vaccine safety and created a targeted goal of improving vaccine safety for the pediatric population (Scheifele \& Halperin 2003).

The sensitivity of a surveillance system is calculated as the proportion of events in a specific population that are actually reported. In lay terms, sensitivity measures the proportion of actual events that are reported to the system. Sensitivity is important to maintain the ability to detect changes over time, which is directly related to the proportion of reports. The need to assess sensitivity has special importance for systems 
that rely on spontaneous reporting. Although higher sensitivity is indicative of a better system in applications such as diagnostic tests, the CDC guidelines state that sensitivity in public health surveillance systems is not directly related to the reporting systems effectiveness. In public health reporting systems, detecting even with low sensitivity is especially important. Low sensitivity may indicate a need for improvement and an inability to respond quickly to reports.

Sensitivity of systems can be improved through changes that allow greater simplicity and timeliness of reporting. The analysis of the sensitivity of the system can be used to evaluate the extent of reporting to the system. Consistent analysis of any surveillance system should be conducted to assure its integrity and assist improvement.

The CDC has performed an analysis of sensitivity of the US Vaccine Adverse Event Reporting System (VAERS) and continues to do so annually (Rosenthal \& Chen 1995; Zhou et al. 2003). Research of the US Vaccine Surveillance System demonstrated that sensitivity varies by AEFI (Goodman \& Nordin 2006). There are no published data on the sensitivity of the CAEFISS. Sensitivity indicates how well a surveillance system functions. By assessing how various factors affect the sensitivity a better understanding will be gained of how certain issues may cause changes in the extent of reporting. These data will be useful to determine ways to improve the Canadian system.

\subsection{Purpose of Study}

The purpose of this study was to assess the sensitivity of the CAEFISS between 1997 and 2008. The study looked at selected vaccines and AEFI based on variation in the indicated population, severity, and incidence to allow for maximum variation and insight into the characteristics of the Canadian system.

\subsection{Specific Aims and Hypotheses}

\subsubsection{Specific Aims}

There were three specific aims. First, this thesis ascertained the sensitivity of the CAEFISS. Second, this study assessed the variability of the sensitivity for selected AEFI over time. This study used the combination of the overall sensitivity and the stability of the sensitivity over time to better understand the characteristics of the system. Third, this study compared AEFI in relation to their rarity, timing of occurrence, and patient age as possible factors affecting sensitivity. 


\subsubsection{Hypotheses}

Based on past studies of sensitivity related to AEFI surveillance systems, existing structures of the Canadian system, and changes of vaccine knowledge over time, the following hypotheses were made:

- Rarer AEFI will have higher sensitivity. This hypothesis parallels the works in assessments of other vaccine adverse event systems and general thoughts on reporting systems found in the literature (Rosenthal \& Chen 1995). The literature clearly indicates that when an $\mathrm{AE}$ is seen more often or known to have a strong correlation, there is a decreased likelihood of reporting (LopezGonzalez et al. 2009).

- AEFI that have a rapid onset will have a higher sensitivity. The sensitivity to events that have a rapid onset will be higher because the time to onset is smaller thus the reporters conclude a temporal association of the event.

- There will be greater sensitivity for AEFI following vaccines given to children as opposed to those given to adults. Pediatric vaccinations will have a higher sensitivity than the adult vaccinations. This hypothesis is based on two factors: first there is greater awareness and experience with vaccines among healthcare providers caring for children, and second, the active surveillance system for adverse events among hospitalized children is conducted by IMPACT (Scheifele \& Halperin 2003). 


\section{CHAPTER 2. LITERATURE REVIEW}

\subsection{Methodology of Search}

A literature review was conducted to locate previous studies addressing the sensitivity of AEFI surveillance systems. A thorough literature review was conducted using both MEDLINE and EMBASE databases from January 01, 1980 through the date of May 31, 2009. A search using the keywords "vaccine", "surveillance systems", "sensitivity", "Canada", and "adverse events" was conducted and articles pulled for relevance. Also websites of key organizations (CDC, PHAC, and the World Health Organization (WHO)) were searched for relevant material on the topic. Secondary to both searches, all papers that were pulled had their references reviewed for possibly relevant articles, and a search for related articles was also conducted. In addition, a search for MeSH terms was done to check the results of the keyword search. The articles were included if they had any relevance related to the assessment of AEFI reporting systems, assessment of reporting systems, sensitivity assessment of AEFI surveillance systems, or assessment of vaccine safety. Inclusion of papers was limited to those written in the English language and studies of humans. All abstracts and relevant papers were pulled for further review to assess their inclusion and relevance.

\subsection{Search Results}

The initial Keyword search using "vaccine", "surveillance systems", "sensitivity", "Canada", and "adverse events" yielded 0 hits; thus, a search excluding the term "Canada" was conducted. This secondary and broader search yielded 4 hits with only one relevant article found: Rosenthal et al. (1995). The search was then expanded utilizing "vaccine" or "adverse event" AND "sensitivity" AND "surveillance systems". After duplicates were eliminated, this third search yielded 20 papers with no newly added relevant articles. The search was again broadened to include "vaccine" and "surveillance system" and yielded 173 unique papers, of which 7 were somewhat relevant to AEFI surveillance systems, 2 looked at sensitivity assessment of the systems, and 1was found in the previous search (Rosenthal \& Chen 1995). These two papers describing sensitivity assessments are discussed in depth in the next sections of this chapter. Other papers had relevant information pertaining to AEFI surveillance systems or vaccine safety in general. To ensure that no articles were missed due to the need to broaden searches and the obvious lack of publications in this area, all relevant papers had their citations searched in attempt to identify relevant articles. No further relevant studies were found in the additional related papers and MeSH term search.

The last portion of the systematic search was the search of the websites of major public health organizations for information. These official websites, especially those of the CDC and WHO, yielded relevant information. For example, the CDC website contained general guidelines for the assessment and maintenance of public health reporting systems 
and, of even greater importance, a discussion of the utilization of sensitivity as an important assessment tool.

\subsection{Assessment of Surveillance Systems}

The systematic search only found one study of relevance to the assessment of sensitivity of a system and one study comparing sensitivities of AEFI surveillance systems. At this time, there are no published studies evaluating the sensitivity rates of CAEFISS.

Rosenthal et al. (1995) conducted the only major study, which assessed the sensitivity of the CDC system in the US between the years of 1985 and 1990 for the United States VAERS. VAERS is the United States' national vaccine surveillance program cosponsored by the CDC and the FDA (Varricchio et al. 2004). The system is a spontaneous safety surveillance system used to report any AEFI in the United States (Iskander, Pool, Zhou, \& English-Bullard 2006; Zhou et al. 2003).

Rosenthal et al. (1995) measured sensitivity for a number of selected vaccines and AEFI pairs. They looked at Oral Polio Vaccine (OPV) - Vaccine Associated Paralytic Polio (VAPP), MMR-seizures, MMR-thrombocytopenia, MMR-rash, and DTP-HHE and DPTseizures. All vaccines and AEFI pairs selected had a proven causal association, as well as an estimated frequency of occurrence. The study used evidence from the published literature to estimate the expected incidence rates of selected AEFI. The number of vaccines doses administered was estimated using: (1) doses purchased with public sector funds and (2) numbers of doses distributed by manufacturers less doses returned by providers (3) the National Health Interview Survey (NHIS) was used for specific childhood vaccinations coverage rate estimates. Using the AEFI rates from the literature, in combination with the estimate of the number of doses administered, the expected number of AEFI occurring was estimated. The number of AEFI reported is divided by the expected number of AEFI to calculate the sensitivity for each specific AEFI. The sensitivity was calculated for overall estimates and separately for only publically administered vaccines. In order for reports to be included as an AEFI they had to meet specific criteria based on timing and age group of the event in reference to the administration. The study found a large range in reporting sensitivity related to the rarity and severity of AEFI. Specifically the sensitivities for reporting poliomyelitis after administration of OPV was $72 \%$ whereas it was less than $1 \%$ for rash and thrombocytopenia after administration of the MMR vaccine. The authors' discussed the issues related to the underreporting of new and rare AEFI, inadequate information reported, and effects of publicity on reporting rates. The authors went to state that sensitivity may also be associated with clinical severity, temporal proximity to vaccination, and healthcare workers' obligations to report particular AEFI. The authors also discussed the need for case-definition that would allow reports to be more efficiently decoded and assessed. Currently the CDC completes the same style analysis annually but does not publish their results.

McNeil et al. (2007) compared the sensitivity of the VAERS system to that of the independently operated US military AEFI Surveillance system. The outcome was used to 
assess the agreement between two systems utilizing a sensitivity value, in which the number of VAERS reports was considered the denominator compared to the number of matching reports in the military system. The analysis was completed with all anthrax related AEFI reports as well as all vaccine reports. The sensitivity of the anthrax only was found to be $74 \%$ and $73 \%$ for all vaccines. In other words, the military AEFI surveillance system picked up on $74 \%$ and $73 \%$ of reports that were also reported to the VAERS. Although the systems are interconnected, the study showed the dynamic nature of many of these AEFI surveillance systems. Moreover, it serves as another example of differences in sensitivity rates based on AEFI, although they referred to the exact same events between systems.

\subsection{Assessing Sensitivity of Surveillance System}

The CDC published a guideline for the analysis of public health reporting system and surveillance systems (German et al. 2001). This guideline applies to all surveillance systems; it discusses many important characteristics that need to be assessed in any system to ensure its quality and dependability of reporting. One of the major factors listed as necessary for assessment of any system is that of sensitivity. The need to assess sensitivity is highly emphasized in the guidelines, especially in systems in which passive reporting is utilized. Low sensitivity may indicate a need for improvement and a lack of ability to respond on time to the reports. The guidelines do add an important note that sensitivity is not directly proportional to how well the system is working. Higher sensitivity of one system may not be equivalent to concluding that one system is performing better than another system.

Even more important than a higher sensitivity is having sensitivity that is high enough to detect changes coupled with stability of the value over time. Sensitivity of systems can be improved through changes that will allow for greater simplicity and timeliness of reporting. The guidelines describe sensitivity as the number of reports collected, divided by the number of reports expected in a specific population under surveillance. The report further emphasizes the need for comparison to literature for expected rates. This parallels the way that sensitivity was analyzed in the Rosenthal et al. (1995) study and this study.

The guidelines discuses how improvement in sensitivity over time can indicate increased awareness, improvements in technology, or can show artifacts necessary for assessment. The guidelines continually state the importance of continuous analysis of any system in place in order to assure its integrity and continuous improvement (German et al. 2001).

\subsection{Assessment of Drug Adverse Event Reporting Systems}

A separate search was conducted for relevant literature related to the assessment of spontaneous AE surveillance systems related to drugs. Literature in this area was much broader than that found related to vaccine topics. There was a separate MeSH term specific for adverse drug reaction surveillance systems but when combined with 
measures of sensitivity there was still a small number of published articles. A further keyword search and related articles search was also conducted to ensure the search was thorough and extensive in nature. The majority of the literature utilizing sensitivity was found to be related to the assessment of hospital or smaller specific systems rather than national or regional systems. A great deal of the recent published literature is related to the creation of signal detection models and applications of various techniques and systems of detection (Handler et al. 2008; Jhung et al. 2007; Koh \& Li 2005; Majdzadeh \& Pourmalek 2008; Nebeker et al. 2007; Visweswaran, Hanbury, Saul, \& Cooper 2003). To further ensure the results from the search were valid, the FDA information service was contacted and the question submitted and there was no internal data or published reports made available related to sensitivity or other sorts of published assessments.

In the studies of smaller systems, the sensitivity is calculated by examining the ratio of the actual number of reports to the true number of events using a gold standard (presumably active) method of surveillance (Betancourt, Hakre, Polyak, \& Pavlin 2007). For example, in a paper published by Hwang et al. (2008) looking at a computer-based adverse event monitoring system, the number of reports picked up by the system was the actual number (numerator) and the number of reports picked up by a pharmacist assessment of the records is noted as the expected (denominator). These two values were then calculated to estimate the sensitivity of their system (Hwang, Lee, Koo, \& Kim 2008). The sensitivity of this computer detection system was found to be $79 \%$.

Few studies had a national level assessment of sensitivity. Studies looking at regional and national reporting in Europe have concluded that the reporting rates would remain around $5 \%$ for all adverse drug reactions nationally (Begaud, Martin, Haramburu, \& Moore 2002). In a study published by McAdams et al. in 2007, the extent of reporting to the FDA adverse event reporting system was estimated for rhabdomyolsis related to statin use (McAdams, Staffa, \& Dal Pan 2008). Their study utilized cohort incidence rate estimates, national distribution estimates of statins, and average day supplies to estimate the number of US statin-associated cases of hospitalized rhabdomyolsis. This 'expected' value was then compared to the observed number of reported cases. This value was referred to as the estimated extent of reporting and was reported separately for every type of statin. This value is calculated similarly to sensitivity but was not referred to as such. The study found that the extent of reporting varied from $5 \%$ to $31.2 \%$ for specific statins. The authors were able to document surveillance system responsiveness to targeted efforts to improve reporting sensitivity. Specifically a mass campaign using "Dear Healthcare Provider" letters led to increased reporting for cerivastatin from $14.8 \%$ to $35.0 \%$.

\subsection{Summary}

Sensitivity has been cited as a useful mechanism of assessing reporting to surveillance systems in much of the literature as well as the CDC guidelines. Despite this noted importance of the assessment of sensitivity, the literature shows a gap in assessments of both drug and AEFI national surveillance systems. Only one study evaluating a national AEFI surveillance system has been published, and was published in 1995. More 
specifically, there is no published work assessing the sensitivity of CAEFISS. Thus this study is unique in that it provides information on the sensitivity of the CAEFISS. The analysis of sensitivity of these systems is helpful in filling an identified gap in the literature and gaining a better understanding of the characteristics of the system in hopes for future improvements. 


\section{CHAPTER 3. METHODS}

\subsection{Overview of Methods}

The methodology used to complete this study can be broken into various steps (Figure 3.1). Each is essential for creating the model used to estimate the values needed to calculate and assess the sensitivity. The model was used to assess which characteristics of the system affect the extent of reporting. The model used to calculate the expected number of AEFI comprises: the published rates of selected AEFI based on proven causal associations, the estimated number of vaccines administered and the calculated expected number of events. Sensitivity was then calculated based on the ratio of observed to expected AEFI reports.

Each of these values can be broken into separate steps and used in the final model and calculations of the sensitivity. The overall sensitivity was calculated for the total study time period, from 1997 through 2008 as well as yearly values within that time range. All of the variables used in the model and calculation were analyzed utilizing Excel.

A descriptive analysis looking at the number of reports in total meeting the criteria as well as by professional affiliation, AEFI type, age groups, and year was conducted and reported. This descriptive analysis gave us an understanding of the variation in the quantity of reports received.

Overall, the selection of the vaccines and their associated AEFI was conducted in a manner that allowed for the greatest variation in age group indication, timing of AEFI, and seriousness related to the AEFI selected. Selection of vaccines and AEFI were based upon the following criteria (Table 3.1):

1) A known correlation between the vaccine and the AEFI.

All of the selected AEFI have been accepted with certainty in the literature to be associated with the specific vaccine. Evidence for correlation was drawn from CDC MMWR Reports, Institute of Medicine (IOM) reports, and past sensitivity studies (Fiore et al. 2009; Rosenthal \& Chen 1995; Stratton et al. 2001).

2) Vaccines routinely administered and recommended to large proportions of the Canadian population.

Newer vaccines (within the last 10 years) and travel vaccines were not included in this study. It is important to note that although newer vaccines were not included some vaccine types may have had newer product lines or changes in formulation during the indicated time period of this study. These were included. 
Step 1 Based on the AEFI and vaccines chosen, a thorough literature search was conducted to find the expected rates of AEFI.

Step 2 PHAC and WHO statistics and survey estimates were used to estimate the Step 2 coverage rates of all vaccines in question over the years of the study.

Step 3 Census Data was used to estimate population sizes.

Step 4 Based on the values developed in Steps 1 -3 a model was created to calculate the expected number of AEFI.

Step 5 Sensitivity was calculated per year and overall. Sensitivity was trended over years in question.

Step 6 One-way and two-way sensitivity analysis of the sensitivity value was conducted.

Figure 3.1 Step-wise Approach to Analysis

Notes: These steps are discussed in full detail in their respective sections in this chapter. PHAC is used to indicate current as well as former activities when the agency was still part of Health Canada. AEFI=Adverse Event Following Immunization; PHAC=Public Health Agency of Canada; WHO=World Health Organization. 
Table 3.1 Vaccines and AEFI to be Analyzed by Temporal Association of AEFI

\begin{tabular}{cccc}
\hline & \multicolumn{3}{c}{ Timing of AEFI } \\
\cline { 2 - 4 } Vaccine & Minutes to Hours & Days & Weeks \\
\hline Influenza & Anaphylaxis & GBS \\
DtaP & Anaphylaxis & HHE & \\
MMR & Anaphylaxis & & Thrombocytopenia \\
Seizures
\end{tabular}

$\mathrm{DTaP}=$ Diphtheria Tetanus and Acellular Pertussis Vaccine; MMR=Measles, Mumps, and Rubella Vaccine; HHE=Hypotonic-Hyporesponsive Episode; GBS=Guillan-Barre Syndrome

3) Analysis of vaccine and AEFI in previous evaluations of surveillance systems (some but not all selected met this criterion) (Rosenthal \& Chen 1995).

These AEFI include seizures, HHE, and thrombocytopenia. Anaphylaxis and GBS were not analyzed in the previous related study.

4) Vaccines used in a variety of age groups.

MMR and DTaP are routinely administered to infants and children versus influenza vaccination which was only analyzed for adults.

5) Variation in timing of AEFI.

Events occurring within hours of vaccine administration include anaphylaxis. Those occurring within days are HHE, severe site reactions, and seizures associated with DTaP those within weeks are GBS associated with influenza vaccination, thrombocytopenia and seizures associated with MMR vaccination.

\subsection{Estimating Expected Rates of AEFI}

In order to derive the expected rates for the AEFI, a systematic literature review was performed using MEDLINE. A search was completed for each vaccine, as well as the AEFI of interest (Table 3.1). A keyword search using the name of the vaccine and the AEFI was conducted. Only English language and human studies were included. Studies retrieved included guidelines, clinical trials, and large epidemiological studies as well as accepted tertiary resources related to AEFI. Acceptable tertiary resources included textbooks and organizational guidelines. All publications used had their citations searched for further relevant literature. When there was clear discordance in published rates efforts were made to select the estimate from the most methodologically sound 
study based on design, power and when it was performed. For each vaccine and AEFI, these published and selected expected rate values were used in the model as the expected incidence of the AEFI.

\subsection{Estimating Expected Number of AEFI}

The next step was to determine the estimated doses of vaccines administered within a time period. This number can only be estimated because Canada does not currently have

a national vaccine registry. An estimated value was derived from the following pieces of information: the number of individuals within an age group indicated to receive the vaccine and the coverage rates for that specific vaccine.

\subsubsection{Population Statistics}

The first component used in the estimates is the population eligible for immunization for a specific vaccine. All population statistics were drawn from Statistics Canada (CANSIM) utilizing the E-STAT online interface (CANSIM : summary directory 2001). Population census data was drawn for every year between 1997-2008 by year and age group. Each vaccine had its indicated population calculated per year. The indicated population can vary per year as the recommendations made by the PHAC change over the years (Canadian immunization guide : user survey : final report 2005). For each year research was done by looking at releases of the Canadian Communicable Disease Report (CCDR) to see if any variations occurred in the age indications of specific vaccines. For the purposes of this study, the annual population for influenza vaccine was all individuals 12 years of age and older. For DTaP, the population comprised children in their first few years of life for the 5 injection series. This is the same population as evaluated for the two injection series for MMR.

\subsubsection{Coverage Rates}

The coverage rate for a vaccine is the proportion of the indicated population who actually received the vaccine in the specified time period. This information can be drawn from published coverage statistics and health surveys conducted by the WHO and PHAC. The WHO/UNICEF review of national immunization coverage is up-dated yearly and reviews many countries immunization rates for various vaccines. For Canada the reports used independent surveys conducted by both UNICEF and the WHO, as well as national surveys conducted by organizations within Canada. The report contains coverage rates for the first DTaP and third DTaP vaccinations from 1987 through to 2008.

For the influenza vaccination coverage rate, the Canadian Community Health Survey (CCHS) was utilized. CCHS is a national health survey conducted every 2-3 years to collect information related to health status, health care utilization, and health determinants of the Canadian population (Beland 2002). Although the survey is designed 
to cover an array of topics related to healthcare, a portion of the survey is used to estimate the influenza vaccination rates for those aged 12 years and above. For intervening years when data were not collected an extrapolated trend value was used to fill the gaps. For example, for 1997, the rate found in the survey was $15 \%$ and in 2000 the survey found a rate of $27 \%$. That suggests there was an increase of $12 \%$ over a 3 year period. This value was then averaged over the 3 years allowing for an annual increase of $4 \%$ in the rate, so that the rate was $19 \%$ and $23 \%$ for 1998 and 1999 respectively.

Lastly, the National Immunization Coverage Survey, which is conducted biannually by PHAC, was used to attain coverage rates. This survey is mailed out nationally to assess immunization coverage rates for 2 and 7 year olds. The survey contains information for four injections of DTaP and the first injection of MMR in those 2 years and younger. It also contains coverage rates of 5 injections of DTaP and 2 injections with MMR for 7 year olds. The survey also contains data regarding the median timing of administration within a window of time. All three data sources were used to determine the best estimate per year of the coverage within the specific indicated population. The resources used for these estimates come from nationally and internationally, credible and non-biased resources. The resources have been used in many published works.

\subsubsection{Estimating Doses Administered and Expected Number of Events}

Using the per year specific estimates of the indicated population and the year-specific estimated coverage rates, the two values were multiplied to calculate the estimated number of doses administered for a year (Equation 3.1). This value was then calculated for each year between 1997 and 2008. The total number of doses administered in that time period was also totaled in order to calculate the total number of doses given in the time period of interest. This value was then used to estimate the total number of events expected (Equation 3.2).

Equation 3.1 Calculation of Estimated Number of Doses Administered:

Estimated Number of Doses Administered $\left(N_{e v}\right) /$ Year $=$ Coverage Rate in specific Year $\left(C_{r}\right) *$ Population Indicated $\left(N_{i}\right)$

Equation 3.2 Calculation of Estimated Number of Events:

\section{Estimated Number of Events/Year $\left(N_{e d}\right)=$ Estimated Number of Doses Administered $\left(N_{d}\right)$} * Event Rate $\left(N_{i}\right)$ 


\subsection{Number of Events and Calculating Sensitivity}

The number of reports was drawn from the CAFISS database. This database has a record of each AEFI reported to the government from consumers, public health, and healthcare providers. All events reported between 1997-2008 were used. The data had no patient identifying information. Specific data obtained for this study was the year vaccine was administered, type of vaccine(s) administered, type of AEFI, age group, and reporter professional affiliation.

The sensitivity value for each AEFI specific for each vaccine was calculated by dividing the number of actual reports by the expected number of reports (Equation 3.3). This value was calculated for each year and overall for the time period in question. The sensitivity trend for each AEFI was compared over time to assess for stability over time. Any reports related to the whole-cell pertussis vaccines and all data from 1997 were excluded to ensure that this transition year did not affect the DTaP sensitivity.

Equation 3.3 Sensitivity Calculation:

Sensitivity $(S)=$ Number of Actual reports $\left(N_{a}\right) /$ Number of Expected Reports $\left(N_{e}\right) * 100 \%$

\subsection{Sensitivity Analysis}

All values used and assumptions of any model are subject to variation and the possibility of error. A sensitivity analysis was used to assess the impact of variations in the assumptions used in the calculations. The first one-way sensitivity was conducted by varying the expected rates used in the model by $+/-5 \%$. A one-way sensitivity analysis was also conducted for the coverage rates assuming a $+/-5 \%$ variance. The two-way analysis was a combination of the one-way analyses in which the variation of the coverage rates and the expected rates of AEFI were investigated simultaneously. This resulted in 4 two-way analyses. 


\section{CHAPTER 4. RESULTS}

\subsection{Expected Rates of AEFI}

Utilizing a through literature review as described in the previous chapter, the expected rates of AEFI and the vaccines of interest were found (Table 4.1). All rates of AEFI rates are reported as the number of cases per number of doses administered. Some AEFI such as GBS with influenza, HHE with DTaP, seizures with MMR and DTaP, and thrombocytopenia with DTaP have known rates that are well documented in the literature and there is a general consensus behind their rates of occurrence. Anaphylaxis with influenza and MMR had rates that greatly varied from each other. Rates were selected from the largest, best-designed studies.

\subsubsection{Influenza}

Influenza vaccination adverse event rates were inconsistent. Anaphylaxis was analyzed for influenza as well as the other two vaccines of interest. Two relevant articles for the rate of anaphylaxis with influenza vaccine were found. One study was a review article (Zent 2002) discussing the possible causes for anaphylaxis and the rate of 0.65 cases per million doses was concluded. The articles go on to state that in general the rate would be around 1 case per million doses administered. The data used was drawn from a VAERS system. The second study was that of Bohlke et al. (2004) which utilized data from HMOs for the pediatric population. They did not report any anaphylaxis cases related to influenza with 197,964 doses administered. They estimated the general risk with any anaphylaxis within the pediatric population was around 0.65 cases per million doses administered. This rate was derived from a general risk of anaphylaxis with any vaccination for the pediatric population. The rate of 1 case per million was selected due to its general consensus in textbooks and review articles for any vaccination (Bohlke et al. 2003; Grabenstein \& Grabenstein 1997; Nokleby 2006). For GBS all studies were in agreement that there is 1 excess case per million doses (Babl, Lewena, \& Brown 2006; Bonhoeffer, Heininger et al. 2004; Juurlink et al. 2006; Lasky et al. 1998; Souayah, Nasar, Suri, \& Qureshi 2007).

\subsubsection{DTaP}

For DTaP, three separate AEFI were assessed. For anaphylaxis the rate with DTaP vaccination was found to be 8.6 cases per million doses (Bohlke et al. 2003). For HHE the accepted rate of occurrence was reported as 140 cases per 140,000 with the acellular pertussis containing vaccine (Babl et al. 2006; Bonhoeffer et al. 2004; Braun et al. 1998; DuVernoy \& Braun 2000). A rate of 0.22 cases of seizure per 1000 doses with DTaP vaccination was selected (Chen, Mootrey, \& DeStefano 2000; Le Saux et al. 2003). 
Table 4.1 Expected Rates of AEFI Based on Literature

\begin{tabular}{|c|c|c|c|}
\hline Vaccine & $\mathrm{AE}$ & $\begin{array}{c}\text { Rates per } \\
\text { Doses } \\
\text { Administered }\end{array}$ & Type of Study/Reason \\
\hline \multirow[t]{2}{*}{ Influenza } & Anaphylaxis & $\begin{array}{l}1 \text { case per } \\
\text { million }\end{array}$ & Review article/consensus \\
\hline & GBS & $\begin{array}{l}1 \text { case per } \\
\text { million }\end{array}$ & Consensus \\
\hline \multirow[t]{3}{*}{$\mathrm{DTaP}$} & Anaphylaxis & $\begin{array}{l}8.6 \text { cases per } \\
\text { million }\end{array}$ & Review/Epidemiological \\
\hline & HHE & $\begin{array}{c}140 \text { cases per } \\
100,000\end{array}$ & Consensus- Epidemiological \\
\hline & Seizures & $\begin{array}{c}0.22 \text { cases per } \\
1000\end{array}$ & Consensus-RCT \\
\hline \multirow[t]{3}{*}{ MMR } & Anaphylaxis & $\begin{array}{l}14.4 \text { per } \\
\text { million }\end{array}$ & Epidemiological \\
\hline & $\begin{array}{l}\text { Thrombocytopenia } \\
\text { (ITP) }\end{array}$ & $\begin{array}{c}1 \text { case in } \\
30,000-40,000\end{array}$ & Consensus- Epidemiological \\
\hline & Seizures & 1 case per 1000 & Consensus-RCT \\
\hline
\end{tabular}




\subsubsection{MMR}

Three AEFI for MMR were assessed. For anaphylaxis, the literature was found to have varying rates. The rate used was 14.4 cases per million doses administered. The rate selected was from a large epidemiological study mentioned above that utilized large HMO data sets to assess the incidence of anaphylaxis (Bohlke et al. 2003). This large dataset has been utilized in prior studies for the vaccine safety datalink and contains approximately 6 million persons worth of data (Chen, DeStefano et al. 2000). Other reported rates, that were not selected, were calculated utilizing surveillance systems. Due to their intrinsic limitation, these systems were not well suited to capture the incidence within a population (Babl, Lewena, \& Brown 2006). One study utilized measure of IgE antibody to attempt to quantify the rate of anaphylaxis (Pool et al. 2002). The study found a rate closer to the concluded rate from the Bohkle et al. (2003) study which was substantially higher that the rates drawn from surveillance systems.

The other two AEFI were thrombocytopenia and seizures both of which have consensus rates that are drawn from both RCTs and epidemiological studies (Babl et al. 2006; Grabenstein \& Grabenstein 1997; Miller et al. 2001; Stowe, Kafatos, Andrews, \& Miller 2008; Stratton, Howe, Johnston Jr, \& Majewski 1994). Thrombocytopenia had a rate of 1 case per 30,000 doses administered and seizures had a rate of 1 case per 1000 doses administered.

\subsection{Estimated Number of Vaccines Administered}

\subsubsection{Coverage Rates}

The estimated number of vaccine doses administered was calculated from the coverage rates (Table 4.2) and the size of the indicated population. It was evident that in both the $\mathrm{DTaP}$ and MMR series coverage rates decreased further into the series. DTaP coverage dropped from an estimate of $97-98 \%$ for the first dose to $85-94 \%$ for the third and $68 \%$ for the $5^{\text {th }}$ dose in the series. MMR had the same trend of a $96 \%$ coverage rate for the first injection in the series throughout most years with a drop to $74 \%$ for the second injection. Influenza vaccination over the years had a general upwards trend in the coverage rate with only 15\% in 1997 and reaching 34\% in 2005-2008 (Figure 4.1).

\subsubsection{Targeted Population and Estimated Doses Administered}

The second value important to the computation of the number of doses administered is that of the size of the indicated population (Table 4.3). There were a total of 42,091,244 doses of DTaP, 12,708,045 doses of MMR, and 90,779,438 doses of influenza vaccines estimated to have been administered between the years of 1997-2008. The table below depicts the numbers attributed to each year as well as the number of individuals who 
Table 4.2 Coverage Rates by Source and Year

\begin{tabular}{|c|c|c|c|c|c|c|c|c|c|c|c|c|c|}
\hline Vaccine & Data Source & 1997 & 1998 & 1999 & 2000 & 2001 & 2002 & 2003 & 2004 & 2005 & 2006 & 2007 & 2008 \\
\hline Influenza & $\begin{array}{c}\text { Canadian } \\
\text { Community } \\
\text { Health Survey }\end{array}$ & $15 \%$ & $19 \% *$ & $23 \% *$ & $27 \%$ & $27 \% *$ & $28 \% *$ & $28 \%$ & $31 \% *$ & $34 \%$ & $34 \% *$ & $34 \% *$ & $34 \% *$ \\
\hline \multirow[t]{5}{*}{ DTaP } & $\begin{array}{l}\text { WHO Estimate } \\
\text { - DTP } 1 \text { Dose }\end{array}$ & $98 \%$ & $98 \%$ & $97 \%$ & $97 \%$ & $97 \%$ & $97 \%$ & $97 \%$ & $97 \%$ & $97 \%$ & $97 \%$ & $97 \%$ & $97 \%$ \\
\hline & $\begin{array}{l}\text { CHS Estimates } \\
\text { - DTP } 2 \text { Dose }\end{array}$ & $92 \%$ & $94 \%$ & $96 \%$ & $94 \%$ & $94 \%$ & $95 \%$ & $94 \%$ & $96 \%$ & $96 \%$ & $96 \%$ & $96 \%$ & $96 \%$ \\
\hline & $\begin{array}{l}\text { WHO Estimate } \\
\text { - DTP } 3 \text { Dose }\end{array}$ & $86 \%$ & $90 \%$ & $94 \%$ & $91 \%$ & $91 \%$ & $93 \%$ & $91 \%$ & $94 \%$ & $94 \%$ & $94 \%$ & $94 \%$ & $94 \%$ \\
\hline & $\begin{array}{c}\text { CHS } 7 \text { year } \\
\text { olds Injection } 4 \\
(2002)\end{array}$ & $75 \%$ & $75 \%$ & $75 \%$ & $75 \%$ & $75 \%$ & $75 \%$ & $75 \%$ & $75 \%$ & $75 \%$ & $75 \%$ & $75 \%$ & $75 \%$ \\
\hline & $\begin{array}{c}\text { CHS -7 year } \\
\text { olds Injection } 5 \\
(2002)\end{array}$ & $68 \%$ & $68 \%$ & $68 \%$ & $68 \%$ & $68 \%$ & $68 \%$ & $68 \%$ & $68 \%$ & $68 \%$ & $68 \%$ & $68 \%$ & $68 \%$ \\
\hline \multirow[t]{2}{*}{ MMR } & $\begin{array}{c}\text { CHS } 7 \text { year } \\
\text { olds Injection } 1 \\
(2002)\end{array}$ & $96 \%$ & $96 \%$ & $96 \%$ & $96 \%$ & $96 \%$ & $96 \%$ & $96 \%$ & $96 \%$ & $96 \%$ & $96 \%$ & $96 \%$ & $96 \%$ \\
\hline & $\begin{array}{c}\text { CHS -7 year } \\
\text { olds Injection } 2 \\
(2002)\end{array}$ & $74 \%$ & $74 \%$ & $74 \%$ & $74 \%$ & $74 \%$ & $74 \%$ & $74 \%$ & $74 \%$ & $74 \%$ & $74 \%$ & $74 \%$ & $74 \%$ \\
\hline
\end{tabular}

Notes: * These values for influenza are extrapolations and no value was available for this year. All values found in multiple sources did not vary by more than $2 \%$ in coverage rate. Rates of influenza are based on the total population aged 12 and older. 


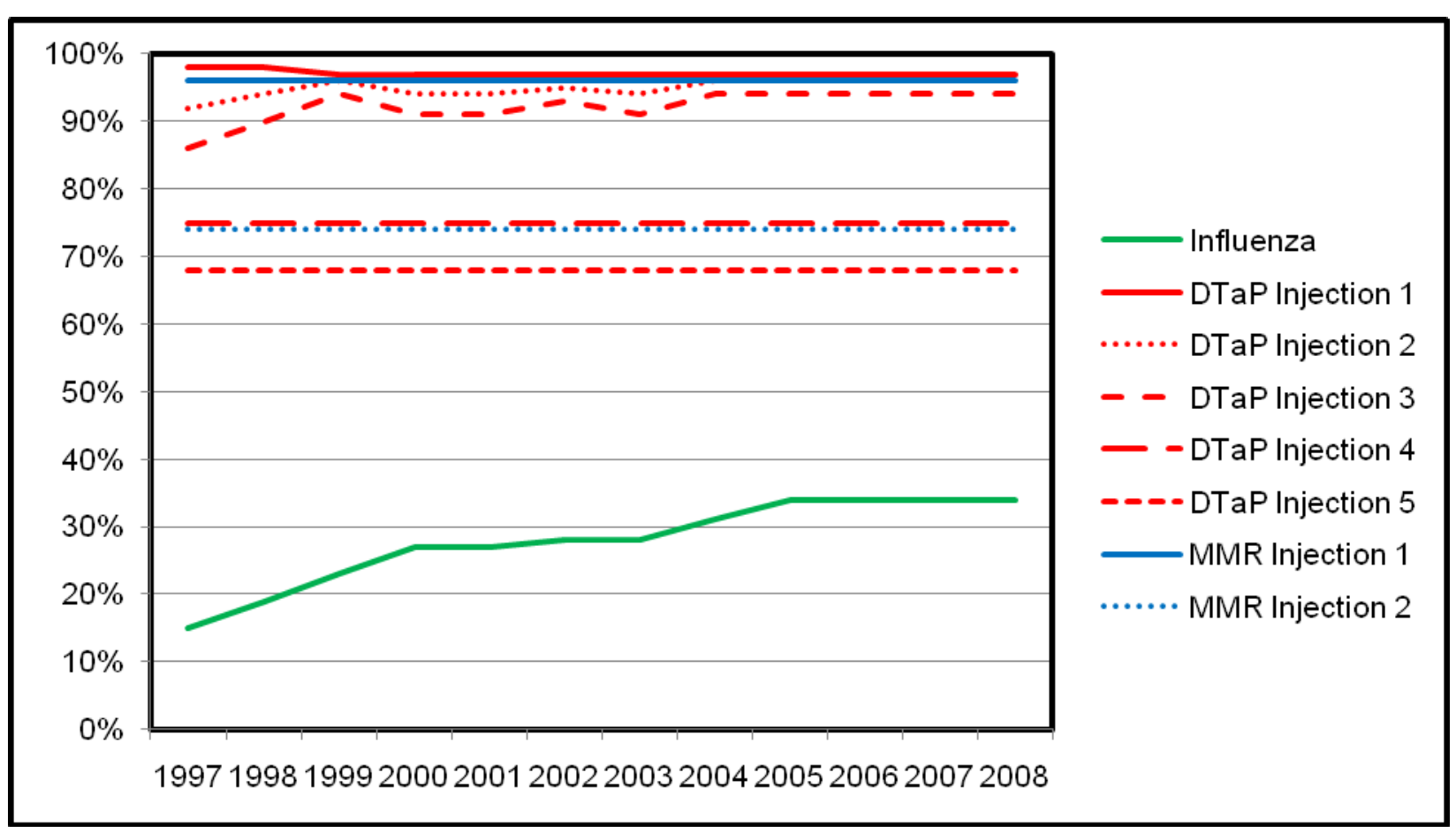

Figure 4.1 Estimated Canadian National Immunization Coverage Rates for Influenza, DTaP, and MMR from 1997-2008 
Table 4.3 Indicated Population and Total Doses per Year from 1997-2008

\begin{tabular}{|c|c|c|c|c|c|c|c|c|c|c|c|}
\hline \multirow[b]{3}{*}{ Year } & \multicolumn{2}{|c|}{ Influenza } & \multicolumn{5}{|c|}{$\mathrm{DTaP}$} & \multicolumn{4}{|c|}{ MMR } \\
\hline & \multirow{2}{*}{$\begin{array}{c}\begin{array}{c}\text { Indicated } \\
\text { Population }\end{array} \\
\begin{array}{c}\text { Seasonal } \\
\text { Injection }\end{array}\end{array}$} & \multirow{2}{*}{$\begin{array}{c}\begin{array}{c}\text { Doses } \\
\text { Administered }\end{array} \\
\text { Total }\end{array}$} & \multicolumn{4}{|c|}{ Indicated Population } & \multirow{2}{*}{$\begin{array}{c}\begin{array}{c}\text { Doses } \\
\text { Administered }\end{array} \\
\text { Total }\end{array}$} & \multicolumn{3}{|c|}{ Indicated Population } & \multirow{2}{*}{$\begin{array}{c}\begin{array}{c}\text { Doses } \\
\text { Administered }\end{array} \\
\text { Total }\end{array}$} \\
\hline & & & $\begin{array}{c}\text { Injections } \\
1-3\end{array}$ & $\begin{array}{c}4^{\text {th }} \\
\text { Injection }\end{array}$ & $\begin{array}{c}\text { 5th } \\
\text { Injection }\end{array}$ & Total & & $\begin{array}{c}1 \mathrm{st} \\
\text { Injection }\end{array}$ & $2^{\text {nd }}$ Injection & Total & \\
\hline 1997 & $25,146,615$ & $3,771,992$ & $1,069,785$ & 381,771 & 408,589 & $1,860,145$ & $1,548,371$ & 381,771 & 387,807 & 769,578 & 653,478 \\
\hline 1998 & $25,427,796$ & $4,831,281$ & $1,034,736$ & 360,180 & 402,518 & $1,797,434$ & $1,516,499$ & 360,180 & 384,062 & 744,242 & 629,979 \\
\hline 1999 & $25,711,987$ & $5,913,757$ & $1,015,290$ & 349,055 & 396,632 & $1,760,977$ & $1,501,103$ & 360,180 & 363,195 & 723,375 & 603,857 \\
\hline 2000 & $26,022,202$ & $7,025,995$ & $1,016,418$ & 343,564 & 393,530 & $1,753,512$ & $1,480,706$ & 343,564 & 353,210 & 696,774 & 591,197 \\
\hline 2001 & $26,391,775$ & $7,125,779$ & 995,583 & 345,751 & 386,870 & $1,728,204$ & $1,458,233$ & 343,564 & 349,540 & 693,104 & 590,581 \\
\hline 2002 & $26,785,043$ & $7,365,887$ & 983,997 & 336,122 & 376,585 & $1,696,704$ & $1,442,967$ & 343,564 & 349,264 & 692,828 & 581,132 \\
\hline 2003 & $27,141,304$ & $7,599,565$ & 990,678 & 332,651 & 364,935 & $1,688,264$ & $1,428,881$ & 343,564 & 339,382 & 682,946 & 570,488 \\
\hline 2004 & $27,498,648$ & $8,524,581$ & $1,014,090$ & 335,567 & 358,767 & $1,708,424$ & $1,464,093$ & 335,567 & 336,889 & 672,456 & 571,442 \\
\hline 2005 & $27,847,352$ & $9,468,100$ & $1,017,642$ & 344,046 & 354,012 & $1,715,700$ & $1,470,611$ & 344,046 & 340,309 & 684,355 & 582,113 \\
\hline 2006 & $28,205,075$ & $9,589,726$ & $1,050,273$ & 345,449 & 351,268 & $1,746,990$ & $1,500,959$ & 345,449 & 348,535 & 693,984 & 589,557 \\
\hline 2007 & $28,576,333$ & $9,715,953$ & $1,069,674$ & 354,142 & 349,395 & $1,773,211$ & $1,524,734$ & 354,142 & 349,049 & 703,191 & 598,273 \\
\hline 2008 & $28,961,242$ & $9,846,822$ & $1,090,365$ & 360,775 & 352,902 & $1,804,042$ & $1,551,853$ & 360,775 & 358,244 & 719,019 & 611,445 \\
\hline Total & $323,715,372$ & $90,779,438$ & $12,348,531$ & $4,189,073$ & $4,496,003$ & $21,033,607$ & $17,889,010$ & $4,189,073$ & $4,259,486$ & $8,448,559$ & $7,173,530$ \\
\hline
\end{tabular}

$\mathrm{DTaP}=$ Diphtheria Tetanus and Acellular Pertussis Vaccine; MMR=Measles, Mumps, and Rubella Vaccine 
were set to receive the doses. The final value found in the table takes into account the coverage rate (Table 4.2).

\subsection{Descriptive Analysis of Reports}

There were 3245 AEFI reports submitted to the CAEFISS between 1997 -2008 (Table 4.4) for the conditions of interest. The distribution of reports varied over the years with 537 reports received in 1997 and only 214 reports received in 2006 . There seems to be no trend of a decline but on average remains steady between 200-300 reports per year. There were 282 reports related to anaphylaxis, 130 for GBS, 575 for HHE, 201 for thrombocytopenia, and 1794 for seizure related AEFI reports (Table 4.5). The greatest number of reports came from nursing related health care professionals with 1454 reports; there were also a large proportion of reports that were from unknown reporters with 1268 reports (Table 4.6).

\subsection{Sensitivity of System}

\subsubsection{Overall Senstivity}

The overall sensitivity for the various AEFI calculated varied greatly (Table 4.7). For influenza the sensitivity was found to be $93.6 \%$ and $136.3 \%$ for GBS and anaphylaxis respectively. For DTaP, sensitivity was found to be $15.0 \%, 1.0 \%$, and $21.2 \%$ for anaphylaxis, HHE, and seizures respectively, and for MMR the rates were $16.5 \%, 52.7 \%$, and $12.7 \%$ in relation to anaphylaxis, thrombocytopenia, and seizures respectively. Sensitivity trends over the years were also analyzed and looked at specifically for each AEFI and vaccine.

\subsubsection{Influenza}

Anaphylaxis related to influenza immunization fluctuated greatly between the years of 1997 and 2008 (Figure 4.2). The lowest rate was found in 2008 with $40.0 \%$ and was found to be as high as $322.2 \%$ in 2004 . Based on the figure it was obvious that there were no general trends but rather a fluctuation in the yearly sensitivity related to anaphylaxis. In analysis of GBS it was found to have a maximum sensitivity of $187.5 \%$ in 2003 and as low as $20.0 \%$ in 1998. Both AEFI exhibited fluctuation year to year. Table 4.4 Descriptive Statistics of Selected Reports from 1997-2008

\subsubsection{DTaP}

Anaphylaxis related to DTaP immunization fluctuated greatly between the years of 1997 and 2008. The highest rate was found in 2008 with $38.5 \%$ and was found to be as low as $0 \%$ in multiple years $(1998,2002$, and 2003). In analysis of HHE it was 
Table 4.4 Descriptive Statistics of Selected Reports from 1997-2008

\begin{tabular}{|c|c|c|}
\hline Year & $\begin{array}{l}\text { Total Number } \\
\text { of Reports Filed }\end{array}$ & $\begin{array}{l}\text { Number of Selected } \\
\text { Reports (N (\%)) }\end{array}$ \\
\hline Total & 46,407 & 3245 \\
\hline 1997 & 4,806 & $537(11.2)$ \\
\hline 1998 & 3,022 & $266(8.8)$ \\
\hline 1999 & 2,956 & $208(7.0)$ \\
\hline 2000 & 5,440 & $245(4.5)$ \\
\hline 2001 & 5,297 & $239(4.5)$ \\
\hline 2002 & 3,886 & $221(5.7)$ \\
\hline 2003 & 3,302 & $220(6.7)$ \\
\hline 2004 & 3,625 & $308(8.5)$ \\
\hline 2005 & 5,831 & $273(4.7)$ \\
\hline 2006 & 4,387 & 214 (4.9) \\
\hline 2007 & 3,855 & $286(7.4)$ \\
\hline $2008 *$ & NA & 228 (NA) \\
\hline
\end{tabular}

Notes: *Selected reports met criteria for selection based on AEFI filing. Total values not available for 2008 . 
Table 4.5 Number of Reports Received by CAEFISS between 1997-2008 Related to Selected Adverse Events by AEFI

\begin{tabular}{cc}
\hline AEFI & $\begin{array}{c}\text { Number of } \\
\text { Reports (N) }\end{array}$ \\
\hline Anaphylaxis & 282 \\
GBS & 130 \\
HHE & 575 \\
Thrombocytopenia & 201 \\
Seizures & 1794 \\
Convulsions & 762 \\
Syncope & 303 \\
Fever & 1055 \\
\hline
\end{tabular}

Table 4.6 Number of Reports Received by CAEFISS between 1997-2008 Related to Selected Adverse Events by Reporters' Affiliation

\begin{tabular}{cc}
\hline Reporter's Affiliation & $\begin{array}{c}\text { Number of } \\
\text { Reports (N) }\end{array}$ \\
\hline Nursing & 1454 \\
Physician & 195 \\
Pharmacist & 7 \\
IMPACT & 129 \\
Manufacturer & 171 \\
Other & 19 \\
Consumer & 1 \\
Unknown & 1268 \\
\hline
\end{tabular}


Table 4.7 Total and Yearly Sensitivity by Vaccine and AEFI for 1997-2008

\begin{tabular}{|c|c|c|c|c|c|c|c|c|c|c|c|c|c|c|c|}
\hline \multirow[b]{3}{*}{ Year } & \multicolumn{6}{|c|}{ Influenza } & \multicolumn{9}{|c|}{ DTaP } \\
\hline & \multicolumn{3}{|c|}{ GBS } & \multicolumn{3}{|c|}{ Anaphylaxis } & \multicolumn{3}{|c|}{ Anaphylaxis } & \multicolumn{3}{|c|}{ HHE } & \multicolumn{3}{|c|}{ Seizures } \\
\hline & $\mathrm{E}$ & $\mathrm{O}$ & Sens & $E$ & $\mathrm{O}$ & Sens & $E$ & $\mathrm{O}$ & Sens & $\mathrm{E}$ & $\mathrm{O}$ & Sens & $\mathrm{E}$ & $\mathrm{O}$ & Sens \\
\hline Overall & 91 & 85 & $93.6 \%$ & 91 & 124 & $136.3 \%$ & 154 & 23 & $15.0 \%$ & 35,778 & 350 & $1.0 \%$ & 3,936 & 835 & $21.2 \%$ \\
\hline $1997^{*}$ & 4 & 2 & $50.0 \%$ & 4 & 5 & $125.0 \%$ & 13 & 1 & $7.7 \%$ & 3,097 & 175 & $5.7 \%$ & 341 & 45 & $13.2 \%$ \\
\hline 1998 & 5 & 1 & $20.0 \%$ & 5 & 5 & $100.0 \%$ & 13 & 0 & $0.0 \%$ & 3,033 & 38 & $1.3 \%$ & 334 & 71 & $21.3 \%$ \\
\hline 1999 & 6 & 6 & $100.0 \%$ & 6 & 6 & $100.0 \%$ & 13 & 1 & $7.7 \%$ & 3,002 & 24 & $0.8 \%$ & 330 & 49 & $14.8 \%$ \\
\hline 2000 & 7 & 6 & $85.7 \%$ & 7 & 13 & $185.7 \%$ & 13 & 1 & $7.7 \%$ & 2,961 & 33 & $1.1 \%$ & 326 & 51 & $15.7 \%$ \\
\hline 2001 & 7 & 6 & $85.7 \%$ & 7 & 13 & $185.7 \%$ & 13 & 2 & $15.4 \%$ & 2,916 & 38 & $1.3 \%$ & 321 & 55 & $17.1 \%$ \\
\hline 2002 & 7 & 12 & $171.4 \%$ & 7 & 14 & $200.0 \%$ & 12 & 0 & $0.0 \%$ & 2,886 & 37 & $1.3 \%$ & 317 & 39 & $12.3 \%$ \\
\hline 2003 & 8 & 15 & $187.5 \%$ & 8 & 5 & $62.5 \%$ & 12 & 0 & $0.0 \%$ & 2,858 & 33 & $1.2 \%$ & 314 & 47 & $15.0 \%$ \\
\hline 2004 & 9 & 13 & $144.4 \%$ & 9 & 29 & $322.2 \%$ & 13 & 4 & $30.8 \%$ & 2,928 & 36 & $1.2 \%$ & 322 & 83 & $25.8 \%$ \\
\hline 2005 & 9 & 4 & $44.4 \%$ & 9 & 10 & $111.1 \%$ & 13 & 4 & $30.8 \%$ & 2,941 & 36 & $1.2 \%$ & 324 & 74 & $22.9 \%$ \\
\hline 2006 & 10 & 6 & $60.0 \%$ & 10 & 5 & $50.0 \%$ & 13 & 2 & $15.4 \%$ & 3,002 & 18 & $0.6 \%$ & 330 & 52 & $15.7 \%$ \\
\hline 2007 & 10 & 6 & $60.0 \%$ & 10 & 15 & $150.0 \%$ & 13 & 4 & $30.8 \%$ & 3,049 & 35 & $1.1 \%$ & 335 & 64 & $19.1 \%$ \\
\hline 2008 & 10 & 8 & $80.0 \%$ & 10 & 4 & $40.0 \%$ & 13 & 5 & $38.5 \%$ & 3,104 & 22 & $0.7 \%$ & 341 & 49 & $14.4 \%$ \\
\hline Mean & & & $90.8 \%$ & & & $136.0 \%$ & & & $15.4 \%$ & & & $1.5 \%$ & & & $17.6 \%$ \\
\hline
\end{tabular}


Table 4.7 Continued

\begin{tabular}{|c|c|c|c|c|c|c|c|c|c|}
\hline \multirow[b]{3}{*}{ Year } & \multicolumn{9}{|c|}{ MMR } \\
\hline & \multicolumn{3}{|c|}{ Anaphylaxis } & \multicolumn{3}{|c|}{ Thrombocytopenia } & \multicolumn{3}{|c|}{ Seizures } \\
\hline & $\mathrm{E}$ & $\mathrm{O}$ & Sens & $\mathrm{E}$ & $\mathrm{O}$ & Sens & $\mathrm{E}$ & $\mathrm{O}$ & Sens \\
\hline Overall & 103 & 17 & $16.5 \%$ & 239 & 126 & $52.7 \%$ & 7,174 & 910 & $12.7 \%$ \\
\hline $1997 *$ & 9 & 2 & $22.2 \%$ & 22 & 10 & $45.5 \%$ & 653 & 127 & $19.4 \%$ \\
\hline 1998 & 9 & 0 & $0.0 \%$ & 21 & 7 & $33.3 \%$ & 630 & 106 & $16.8 \%$ \\
\hline 1999 & 9 & 0 & $0.0 \%$ & 20 & 16 & $80.0 \%$ & 604 & 74 & $12.3 \%$ \\
\hline 2000 & 9 & 0 & $0.0 \%$ & 20 & 16 & $80.0 \%$ & 591 & 63 & $10.7 \%$ \\
\hline 2001 & 9 & 1 & $11.1 \%$ & 20 & 10 & $50.0 \%$ & 591 & 58 & $9.8 \%$ \\
\hline 2002 & 8 & 0 & $0.0 \%$ & 19 & 8 & $42.1 \%$ & 581 & 44 & $7.6 \%$ \\
\hline 2003 & 8 & 1 & $12.5 \%$ & 19 & 5 & $26.3 \%$ & 570 & 47 & $8.2 \%$ \\
\hline 2004 & 8 & 3 & $37.5 \%$ & 19 & 10 & $52.6 \%$ & 571 & 62 & $10.9 \%$ \\
\hline 2005 & 8 & 1 & $12.5 \%$ & 19 & 11 & $57.9 \%$ & 582 & 72 & $12.4 \%$ \\
\hline 2006 & 8 & 1 & $12.5 \%$ & 20 & 11 & $55.0 \%$ & 590 & 70 & $11.9 \%$ \\
\hline 2007 & 9 & 4 & $44.4 \%$ & 20 & 17 & $85.0 \%$ & 598 & 85 & $14.2 \%$ \\
\hline 2008 & 9 & 4 & $44.4 \%$ & 20 & 6 & $30.0 \%$ & 611 & 71 & $11.6 \%$ \\
\hline Mean & & & $16.4 \%$ & & & $53.1 \%$ & & & $12.1 \%$ \\
\hline
\end{tabular}

$\mathrm{E}=$ Expected; $\mathrm{O}=$ Observed; Sens=Sensitivity; $\mathrm{DTaP}=$ Diphtheria Tetanus and Acellular Pertussis Vaccine; $\mathrm{MMR}=\mathrm{Measles}$, Mumps, and Rubella Vaccine; HHE=Hypotonic-Hyporesponsive Episode; GBS=Guillan-Barre Syndrome

Note: For DTaP Values for the year of 1997 were not included in the overall and the mean values due to the change from the whole cellular to acellular pertussis in that year. 


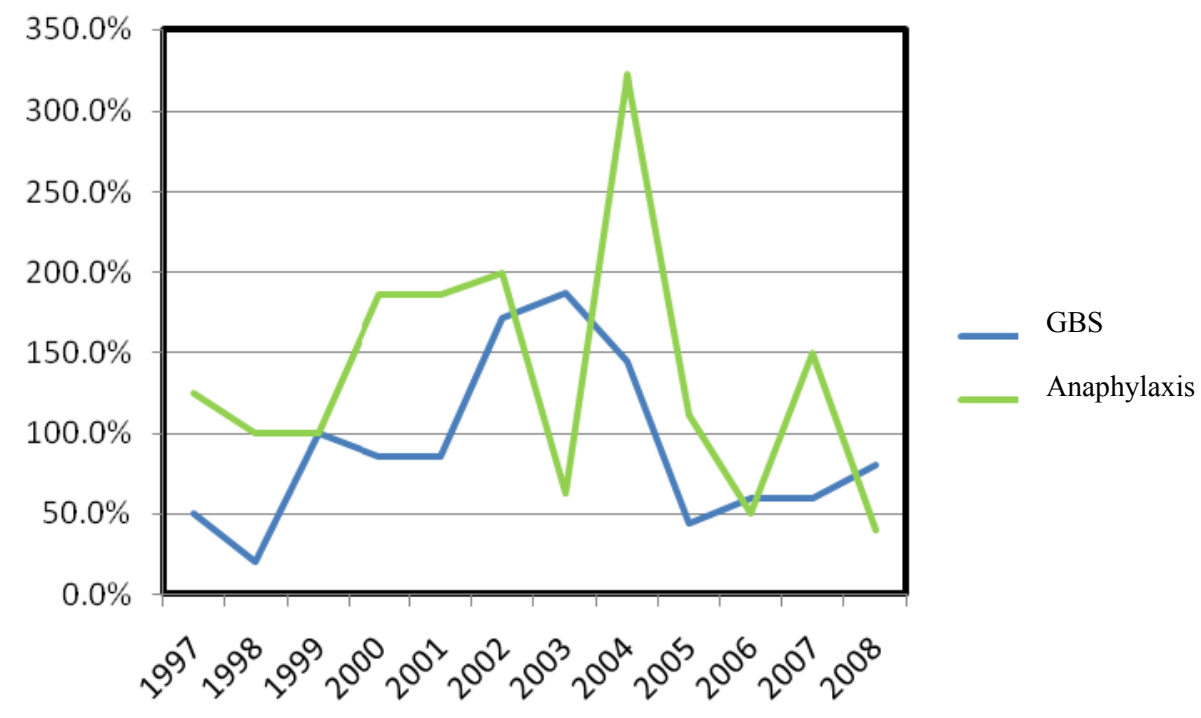

Figure 4.2 Sensitivity for Anaphylaxis and GBS Related to Influenza Immunizations

found to have a maximum sensitivity of $5.7 \%$ in 1997 and as low as $0.6 \%$ in 2006 . There was a stable level of reporting across time with little fluctuation of the sensitivity value. Seizures had the highest sensitivity in 2004 with $25.8 \%$ and the lowest in 2002 with 12.3\% (Figure 4.3).

\subsubsection{MMR}

Anaphylaxis related to MMR immunization fluctuated greatly between the years of 1997 and 2008 (Figure 4.4). The highest rate was found in 2008 with $44.4 \%$ and was found to be as low as $0 \%$ in several years (1998-2000). In analysis of Thrombocytopenia it was found to have a maximum sensitivity of $85 \%$ in 2007 and as low as $26.3 \%$ in 2003 . Seizures had the highest sensitivity in 1997 with $19.4 \%$ and the lowest in 2002 with $7.6 \%$.

\subsection{Sensitivity Analysis}

A sensitivity analysis was conducted to assess the level of variation in the total sensitivity of the system based on the variation in the expected event rate $(+/-5 \%)$ and total number of administered doses (+/- 5\%). A two-way analysis was also conducted using both variations of the doses and $+/-5 \%$ of the event rate to create four different permutations. The difference from the primary model and the sensitivity analysis value was reported. For DTaP anaphylaxis ranged from $10.5 \%$ to $11.6 \%$, HHE ranged from $0.6 \%$ to $0.7 \%$, and seizures ranged from $8.9 \%$ to $10.3 \%$. 


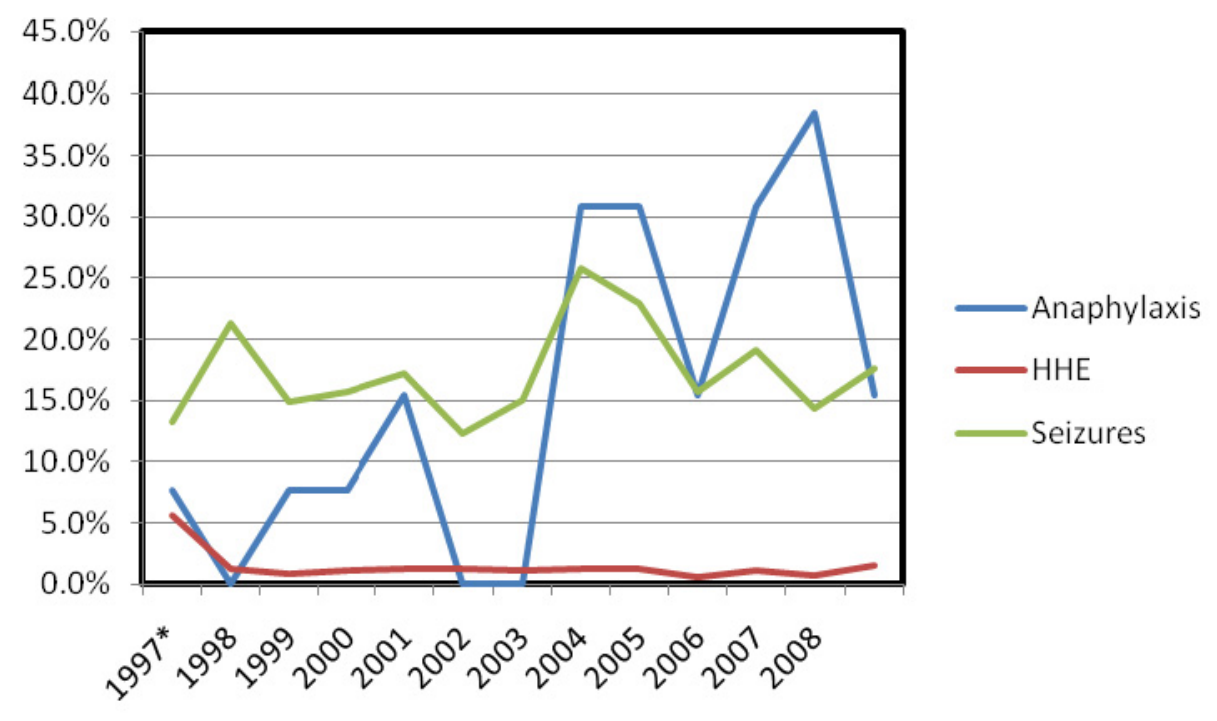

Figure 4.3 Sensitivity for anaphylaxis, HHE, and Seizures Related to DTaP Immunizations

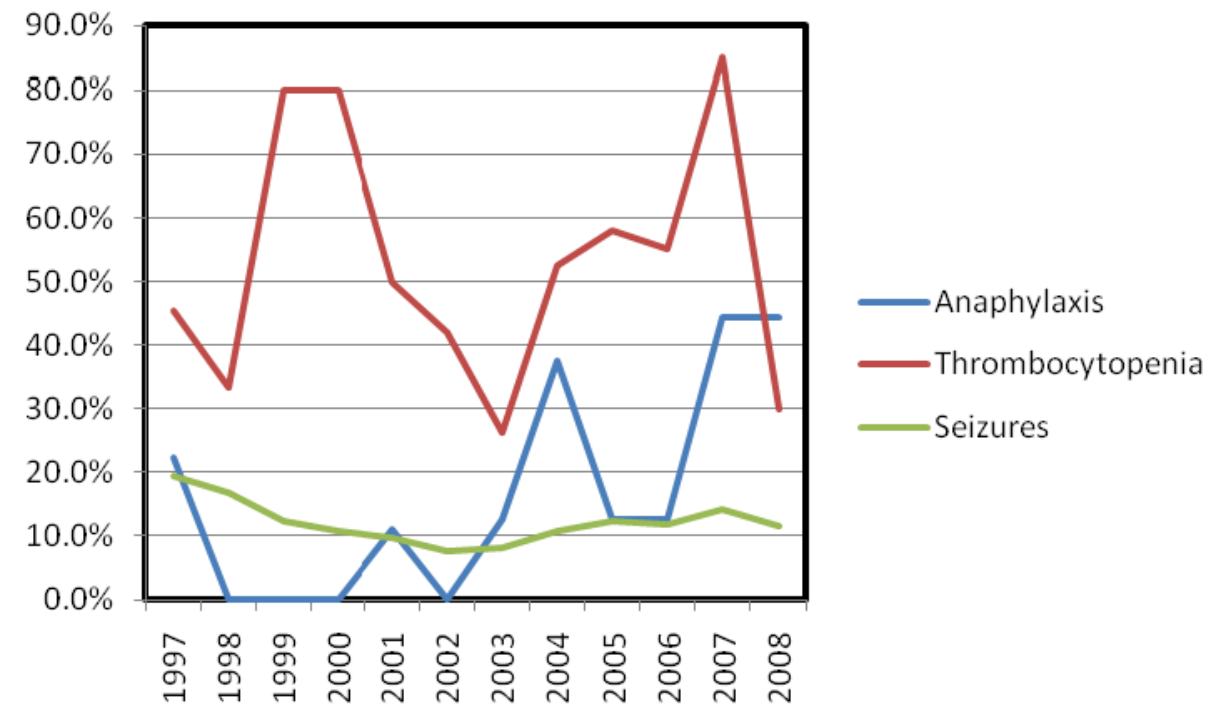

Figure 4.4 Sensitivity for Anaphylaxis, Thrombocytopenia, and Seizures Related to MMR Immunizations 
For MMR, anaphylaxis ranged $12.0 \%$ to $14.0 \%$. There was a greater total fluctuation with the rarer AEFI such as anaphylaxis, GBS, and thrombocytopenia (Table 4.8). 
Table 4.8 One- and Two-way Sensitivity Analysis

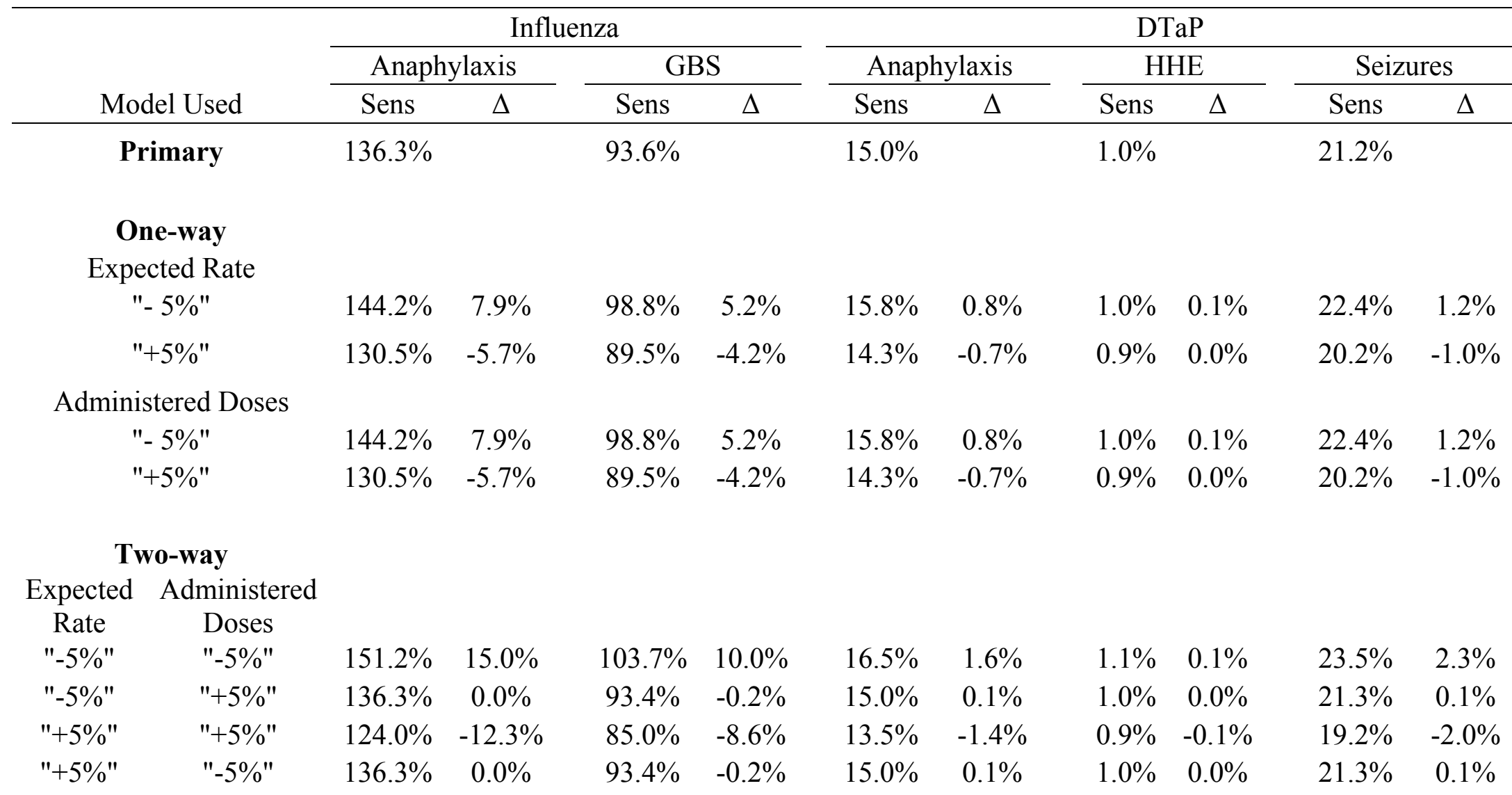


Table 4.8 Continued

\begin{tabular}{|c|c|c|c|c|c|c|}
\hline \multirow[b]{3}{*}{ Model Used } & \multicolumn{6}{|c|}{ MMR } \\
\hline & \multicolumn{2}{|c|}{ Anaphylaxis } & \multicolumn{2}{|c|}{ Thrombocytopenia } & \multicolumn{2}{|c|}{ Seizures } \\
\hline & Sens & $\Delta$ & Sens & $\Delta$ & Sens & $\Delta$ \\
\hline Primary & $16.5 \%$ & & $52.7 \%$ & & $12.7 \%$ & \\
\hline \multicolumn{7}{|l|}{ One-way } \\
\hline \multicolumn{7}{|l|}{ Expected Rate } \\
\hline "- 5\%" & $17.3 \%$ & $0.9 \%$ & $55.5 \%$ & $2.8 \%$ & $13.4 \%$ & $0.7 \%$ \\
\hline$"+5 \% "$ & $15.7 \%$ & $-0.7 \%$ & $50.2 \%$ & $-2.5 \%$ & $12.1 \%$ & $-0.6 \%$ \\
\hline \multicolumn{7}{|c|}{ Administered Doses } \\
\hline "- 5\%" & $17.3 \%$ & $0.9 \%$ & $55.5 \%$ & $2.8 \%$ & $13.4 \%$ & $0.7 \%$ \\
\hline "+5\%" & $15.7 \%$ & $-0.7 \%$ & $50.2 \%$ & $-2.5 \%$ & $12.1 \%$ & $-0.6 \%$ \\
\hline
\end{tabular}

\begin{tabular}{cccccccc}
\multicolumn{2}{c}{ Two-way } \\
$\begin{array}{ccccccc}\text { Expected } \\
\text { Rate }\end{array}$ & $\begin{array}{c}\text { Administered } \\
\text { Doses }\end{array}$ & & & & & & \\
$"-5 \% "$ & $"-5 \% "$ & $18.3 \%$ & $1.8 \%$ & $58.3 \%$ & $5.6 \%$ & $14.1 \%$ & $1.4 \%$ \\
$"-5 \% "$ & $"+5 \% "$ & $16.5 \%$ & $0.0 \%$ & $52.7 \%$ & $0.0 \%$ & $12.7 \%$ & $0.0 \%$ \\
$"+5 \% "$ & $"+5 \% "$ & $14.9 \%$ & $-1.5 \%$ & $47.7 \%$ & $-5.0 \%$ & $11.5 \%$ & $-1.2 \%$ \\
$"+5 \% "$ & $"-5 \% "$ & $16.5 \%$ & $0.0 \%$ & $52.7 \%$ & $0.0 \%$ & $12.7 \%$ & $0.0 \%$ \\
\hline
\end{tabular}

Sens $=$ Sensitivity, $\Delta=$ Difference between Primary model and sensitivity analysis value; DTaP=Diphtheria Tetanus and Acellular Pertussis Vaccine; MMR=Measles, Mumps, and Rubella Vaccine; HHE=Hypotonic-Hyporesponsive Episode; GBS $=$ Guillan-Barre Syndrome 


\section{CHAPTER 5. DISCUSSION}

\subsection{Overview of Findings}

This is the first study to assess the sensitivity of the CAEFISS. Sensitivity varied by vaccines and within vaccines specific AEFI, from 1997 to 2008. The overall sensitivity varied by selected AEFI from $1.0 \%$ to $136.3 \%$. The greatest variations were found in anaphylaxis which was analyzed in all three of the vaccines examined. Variations in sensitivity were found between vaccines, AEFI within the same vaccine and over within and among vaccines. There was evidence that age group, timing of event, and rarity also affected sensitivity within vaccines.

\subsubsection{Comparison to Past Literature}

As discussed in Chapter 2, the only major study to look at the assessment of sensitivity of a vaccine AEFI surveillance system was that of Rosenthal et al. (1995). This study had four similar AEFI that overlapped with those in the paper by Rosenthal et al. (1995): seizures associated with DTaP and MMR, HHE with DTaP, and thrombocytopenia with MMR.

In comparing thrombocytopenia associated with MMR, the sensitivity was less than one percent in the study by Rosenthal et al. (1995) and 52.7\% in the present study. This difference may be explained in large part by the introduction of the IMPACT program and its specific targeting of thrombocytopenia through the use of the active surveillance system (Jadavji, Scheifele, \& Halperin 2003).

Sensitivity for seizures related to DTaP was higher in the study by Rosenthal et al. (1995) than in the present study. Rosenthal et al. (1995) found sensitivity values of $24 \%$ versus $17.6 \%$ in this study. While these differences are significant, note that a whole-cell pertussis vaccine known to be associated with a higher expected rate of seizures was used during the time period that Rosenthal analyzed. The vaccine used during the course of this study is acellular with a lower of incidence of seizure as discussed previously. This risk of seizures was of a greater concern to clinicians during that time period than during the period studied here, so it was more likely that clinicians would report its appearance. This has become less of a concern as a result of the formulation change, a downward trend of reporting by clinicians and the public was expected. The present study excluded any reports related to the whole-cell pertussis vaccines, and for the overall value calculation, all data from 1997 were excluded to ensure that this transition year did not affect the sensitivity.

Sensitivity for seizures related to MMR was also higher in the study by Rosenthal et al. (1995) than in the present study. Rosenthal and his colleagues found sensitivity values of $27 \%$ versus $12.1 \%$ in this current study. This may be related to the correlation between DTaP and MMR. As discussed above, the concern of clinicians at the time of the 
Rosenthal study for seizures was much higher than recent years.MMR and DTaP administration are closely related and often administered at similar visits. There were a large proportion of reports of seizures in this study in which both DTaP and MMR were both reported to have been administered.

This study also found lower sensitivity associated with HHE (approximately $4 \%$ versus $1.0 \%$ in our study). This difference may be associated with a general downward trend in reporting. This was expected, because as a vaccine is used more often, clinicians become more acclimated to its use and associated AEFI leading to fewer reports.

Although the results varied, Rosenthal and colleagues made similar conclusions with what specific factors affected sensitivity, such as clinical seriousness, temporal proximity, and healthcare awareness. These findings paralleled this study's finding that sensitivity was affected by both temporal proximity and expected rates of occurrence. The differences of results may be due to variations in the systems and timing of the studies. The time period in which their analysis was conducted (1985-1990) differed from that in this study (1997-2008).

\subsubsection{Results by Vaccines}

\subsubsection{AEFI Related to Influenza Vaccination}

In the present study, influenza vaccination was used as an indicator of all adult immunizations. Estimates of sensitivity for influenza vaccine-associated reporting of anaphylaxis and GBS were limited to those aged 12 years and older. The highest adult sensitivities were for GBS and anaphylaxis (136.3\% and 93.6\%, respectively). It was evident that the immunization of adults with influenza vaccine had the highest sensitivity in the study.

Sensitivity of both GBS and anaphylaxis related to influenza vaccination were highly unstable when analyzed on a yearly basis. This variation may be due to the rarity of the events. This rarity led to a small number of expected events which made small variations in the actual data appear more significant than they truly were in the overall sensitivity. This could be even more pronounced when other factors within the population impact the occurrence of an AEFI. One example of this confounding is the occurrence of GBS due to an outbreak of Campylobacter.

Other factors such as the rate of occurrence and the assumption that all cases are true cases could also impact these rare adverse events. Rare events have an increased likelihood of being misreported because reporters may be less experienced with a rare AEFI. This lack of experience may also increase the likelihood of misreporting and misdiagnosing adverse events, leading to inconsistency in reporting. This inconsistency is even more pronounced without case-definitions. Without the assistance of case definitions the literature has shown clinicians' case determinations are inconsistent (Kohl et al. 2008). Inconsistency in reporting may be one of the major causes of greater 
variation in reporting, and this effect would be much more pronounced for adverse events with low rates of occurrence.

\subsubsection{AEFI Related to DTaP Vaccination}

Estimates of sensitivity for DTaP related AEFI were completed for anaphylaxis, HHE, and seizures. The overall sensitivity was found to be $15.0 \%$ for anaphylaxis, $1.0 \%$ for thrombocytopenia, and $21.2 \%$ for seizures.

Seizures had the highest sensitivity of the three AEFI related to DTaP. The higher reporting of seizures maybe due to the past experience with the whole-cell pertussis vaccine, which was changed to the acellular formulation due to concerns with the rate of seizures related to vaccination. Although the rate of occurrence of the AEFI was decreased, this may still be an area of concern for clinicians. Seizures are also a relatively more common adverse event and displayed a steady sensitivity over time.

HHE was the most common of the three AEFI and exhibited the lowest sensitivity. HHE was the most stable sensitivity of all the AEFI in the study and within DTaP related AEFI. This is most likely due to HHE having the highest rate of occurrence for DTaP AEFI and AEFI in the study as a whole. Sensitivity for HHE and seizures were stable over the years.

Anaphylaxis related to DTaP vaccination was not the highest sensitivity within DTaP. This varies from the other two vaccines looked at in the study, in which anaphylaxis was the highest of the AEFI within vaccines. The results of anaphylaxis within DTaP did parallel those in the other vaccines in terms of year to year sensitivity, which was found to vary greatly.

\subsubsection{AEFI Related to MMR Vaccination}

Estimates of sensitivity for MMR related AEFI were completed for anaphylaxis, thrombocytopenia, and seizures. The overall sensitivity was found to be $16.5 \%$ for anaphylaxis, $52.7 \%$ for thrombocytopenia, and $12.7 \%$ for seizures.

Thrombocytopenia was found to have the highest sensitivity of the three AEFI related to MMR. This may be explained by the IMPACT program and its specific targeting of thrombocytopenia through the use of the active surveillance system (Jadavji et al. 2003). It may be difficult to determine how much of this is due to the IMPACT program. Only 33 out of the 239 thrombocytopenia reports were coded as IMPACT, some reports may be related to the program and labeled as reports from nurses or physicians.

Seizures were the most common of the three AEFI and exhibited the lowest sensitivity. Sensitivity for seizures was stable over the years when compared to thrombocytopenia and anaphylaxis. This variation of anaphylaxis and thrombocytopenia may be due to the rarity of the events. 


\subsubsection{Adult versus Pediatric Reporting}

In the present study, influenza vaccination was used as an indicator of adult immunizations. DTaP and MMR were predominantly used as indicators of immunization in the pediatric population. Estimates of sensitivity for influenza vaccine-associated reporting of anaphylaxis and GBS were limited to those aged 12 years and older, whereas DTaP and MMR sensitivity were limited to preschool-aged populations to the age of 8 years. The highest adult sensitivities were for GBS and anaphylaxis. There was evidence that the immunization of adults with influenza vaccine had a higher sensitivity. It is important to note that the AEFI selected for influenza were both rare and this may limit the ability to conclude that adult sensitivity was higher than that of the pediatric population.

This differentiation of adult immunization and pediatric immunization may be attributed to factors pertaining to the individual responsible for reporting and administering the vaccines (Duclos, Hockin, Pless, \& Lawlor 1997). Further research would be required to help better understand these findings.

\subsubsection{Common versus Rare Adverse Events}

The AEFI considered within this study varied in rate of occurrence with some being more common than others. As discussed in the Methods chapter, these vaccines were selected on the basis of the variation in their expected rates of occurrence in order to compare more common AEFI with rarer AEFI (Table 5.1). The event with the highest expected rate of occurrence was HHE associated with DTaP. This adverse event was also associated with the lowest overall sensitivity rate in this study. Seizures with both MMR and DTaP had lower sensitivity of $12.7 \%$ and $21.2 \%$, respectively. They were the $2^{\text {nd }}$ and $3^{\text {rd }}$ lowest sensitivity values found. In contrast, the three most common adverse events were among the lowest sensitivity values of the study and exhibited the greatest stability from year to year.

AEFI that have higher rates of occurrence appear to have a lower sensitivity and more stable sensitivity over time. As an AEFI becomes more common, it will be more frequently seen by those administering the vaccine, and its sensitivity may decrease and the stability of reporting may increase. Rarer events have a greater likelihood that they will be misdiagnosed than more common events. This misdiagnosis may be due to a lack of familiarity with the event because of its rarity.

As discussed above, inconsistencies in reporting may also affect the stability of sensitivity over time and are more pronounced in rare adverse events. Rare AEFI may also be more greatly affected by smaller variations in their actual occurrence. A greater number of reports would be needed to vary the sensitivity of a common AEFI with a higher rate of occurrence. Rarer adverse events had the greatest variations when estimates were changed in the model. 
Table 5.1 Ordered Expected Occurrence per Million Doses from Lowest to Highest

\begin{tabular}{|c|c|c|c|c|c|}
\hline Order & $\begin{array}{l}\text { Adverse Event } \\
\text { (Vaccine) }\end{array}$ & $\begin{array}{c}\text { Rate of } \\
\text { Occurrence } \\
\text { per Million } \\
\text { Doses }\end{array}$ & $\begin{array}{c}\text { Overall } \\
\text { Sensitivity }\end{array}$ & $\begin{array}{l}\text { Average } \\
\text { Sensitivity }\end{array}$ & $\begin{array}{c}\text { Sensitivity } \\
\text { Range }\end{array}$ \\
\hline 1 & HHE (DTaP) & 14000 & $1.0 \%$ & $1.5 \%$ & $\begin{array}{l}0.6 \%- \\
1.3 \%\end{array}$ \\
\hline 2 & Seizures (MMR) & 1000 & $12.7 \%$ & $12.1 \%$ & $\begin{array}{l}7.6 \%- \\
19.4 \%\end{array}$ \\
\hline 3 & Seizures (DTaP) & 220 & $21.2 \%$ & $17.6 \%$ & $\begin{array}{l}12.3 \%- \\
25.8 \%\end{array}$ \\
\hline 4 & $\begin{array}{l}\text { Thrombocytopenia } \\
\text { (MMR) }\end{array}$ & 33 & $52.7 \%$ & $53.1 \%$ & $\begin{array}{l}26.3 \%- \\
85.0 \%\end{array}$ \\
\hline 5 & $\begin{array}{c}\text { Anaphylaxis } \\
\text { (MMR) }\end{array}$ & 14 & $16.5 \%$ & $16.4 \%$ & $\begin{array}{l}0.0 \%- \\
44.4 \%\end{array}$ \\
\hline 6 & $\begin{array}{l}\text { Anaphylaxis } \\
\text { (DTaP) }\end{array}$ & 9 & $15.0 \%$ & $15.4 \%$ & $\begin{array}{l}0.0 \%- \\
38,5 \%\end{array}$ \\
\hline 7 & $\begin{array}{l}\text { Anaphylaxis } \\
\text { (Influenza) }\end{array}$ & 1 & $93.6 \%$ & $136.0 \%$ & $\begin{array}{l}40.0 \%- \\
322.2 \%\end{array}$ \\
\hline 8 & GBS (Influenza) & 1 & $136.3 \%$ & $90.8 \%$ & $\begin{array}{l}20.0 \%- \\
187.5 \%\end{array}$ \\
\hline
\end{tabular}

\subsubsection{Timing of AEFI Onset}

For all three of the vaccines analyzed, the most acute AEFI assessed was anaphylaxis, a serious systemic reaction that sometimes occurs in response to any vaccine and commonly occurs within minutes to hours after administration (Coop et al. 2008; Erlewyn-Lajeunesse, Bonhoeffer, Ruggeberg, \& Heath 2007; Kagy \& Blaiss 1998). Anaphylaxis related to influenza vaccination was found to have the highest sensitivity in the study, with an overall sensitivity of $136.6 \%$. This value was the only value in the study that was found to be above $100 \%$, meaning that there was more reporting than expected. This high sensitivity may, in part, be due to over-reporting or a misdiagnosis of the adverse event.

AEFI with slower onsets of days to weeks had lower sensitivities. GBS related to influenza vaccination, seizures related to MMR vaccination, and HHE related to DTaP were the selected to represent AEFI that have a longer duration of onset post immunizations. These AEFI were also the lowest sensitivities in their respective vaccines. This may show that when an AEFI is temporally separated from the administration of the vaccine, it is less likely to be reported. This may be due to the more difficult task of finding correlation to the vaccine days or weeks after its administration, as compared to minutes and hours after its administration. 
In addition to being temporally acute, anaphylaxis is also a rare AEFI as discussed previously. Examination of trends over years for all three vaccines showed that anaphylaxis was present with the greatest variations over time. Thus, not only does the temporal acuity of an adverse event seem to increase the reporting, but it also may be a factor in determining the instability of reporting over time. This may also be clouded by the general rarity of anaphylaxis.

\subsubsection{Sensitivity Analysis}

A sensitivity analysis of this study was conducted to assess the level of variation in the overall sensitivity. One-way and two-way analyses were conducted for the two major values in the model: the expected rate and the number of administered doses. The greatest variation was seen with the rare adverse events. GBS and anaphylaxis related to influenza vaccination had a variation of $10.0 \%$ and $15.0 \%$, respectively. The most common adverse events such as HHE and seizures related to DTaP had variations of $0.1 \%$ and $2.3 \%$, respectively. This can be attributed to the fact that a single additional case can cause great variation in the calculated sensitivity of rare adverse events, compared to the more common adverse events. The results from the sensitivity analysis can be used to better determine how much credence to lend to these results. It can be assumed that the specific findings of the more common adverse events are more robust. In contrast, those rare adverse events need greater scrutiny and more complex analysis when large changes in sensitivity occur. This adds increased importance for the need to standardize reporting through standard case definitions, in order to stabilize the sensitivity. Therefore when large variations in reports occur, there can be a quicker response to the number of reports, rather than a need for further assessment into the PPV of the reports. Reporters will be diligent in reporting cases that meet a level of diagnostic certainty as comfort with casedefinitions increases.

\subsection{Limitations}

\subsubsection{Expected Rates of AEFI}

Expected rates had to be estimated based on previous clinical trials and epidemiological studies, which may not produce perfect expected rates. In order to address this study limitation, a thorough systematic search was conducted using multiple sources. In situations where multiple values were found, the best designed studies were used as the primary value. Additionally, to ensure that values were robust, a sensitivity analysis of this study was utilized to assess how greatly the variation in the expected rates would affect the results. The variation in the expected rate affected the rarer adverse events to a great degree, as discussed previously. The greatest fluctuation was seen in anaphylaxis associated with influenza vaccination. It varied from $124.0 \%$ to $151.2 \%$. In more common adverse events such as HHE, the variation due to these estimates was far less pronounced, varying by only $0.1 \%$. 


\subsubsection{Coverage Rates}

Coverage rates were used in the model to compute the expected number of administered doses. As discussed in past chapters, these values as discussed in past chapters were drawn from survey data. In some of the cases of DTaP, MMR, and influenza the surveys were not conducted yearly. In years where there were no surveys completed, an extrapolation of the past and future coverage rates was used, assuming a linear growth. This assumption may not be valid as there may be unknown but important factors affecting the coverage rate. This may not be as distinct in DTaP and MMR vaccinations since their rates for all the doses held within $1-2 \%$ of each other throughout the time period. The greatest impact this may have is in the influenza vaccination which had a great increase in rate over the time period analyzed. To ensure that our estimations were not unrealistic a double check was conducted using the number of influenza doses distributed nationally. Dose distribution data were provided by PHAC who received the data from the manufacturers. It was found that estimates for doses administered were reasonably close to these values (Table 5.2). The estimates never exceeded the number of doses distributed. To also insure that estimates did not highly impact the final values as a part of the sensitivity analysis conducted all coverage rates were adjusted by $+/-5 \%$ for both the one-way analysis and two-way analysis. It was found that this adjustment had the greatest impact on the rarer adverse events similar to the results found with the expected rates.

Table 5.2 Comparison of Estimated Influenza Vaccine Doses Administered to Reported Doses Distributed

\begin{tabular}{ccc}
\hline Year & $\begin{array}{c}\text { Estimated Doses } \\
\text { Administered }\end{array}$ & Doses Distributed \\
\hline Overall & $90,779,438$ & $115,824,359$ \\
1997 & $3,771,992$ & $5,249,999$ \\
1998 & $4,831,281$ & $5,062,361$ \\
1999 & $5,913,757$ & $5,750,237$ \\
2000 & $7,025,995$ & $12,199,380$ \\
2001 & $7,125,779$ & $9,330,555$ \\
2002 & $7,365,887$ & $10,477,216$ \\
2003 & $7,599,565$ & $11,080,935$ \\
2004 & $8,524,581$ & $11,309,614$ \\
2005 & $9,468,100$ & $11,494,459$ \\
2006 & $9,589,726$ & $11,893,503$ \\
2007 & $9,715,953$ & $11,443,640$ \\
2008 & $9,846,822$ & $10,532,460$ \\
\hline
\end{tabular}

Note: These numbers are for total doses of all types of influenza vaccine marketed in Canada 


\subsubsection{Issues with Spontaneous Systems}

One Typical key limitation in the use of spontaneous reporting system data is missing and incomplete reports. This inconsistency in data makes it difficult to analyze many of the reports since they may not contain all the necessary data. This limitation does not impact our study, because the information was limited to age of patient, year of report, vaccines administered, and type of adverse event which are almost always present within a report.

Another limitation with reports associated with spontaneous systems is the PPV of the reports and how this may affect the sensitivity. Two factors can affect whether a report represents a true adverse event causally related to the vaccine. The first factor is that of the factuality of the report. In other words, is the report of a specific AEFI truly related to that AEFI, or is it a misdiagnosis? For anaphylaxis this is especially problematic since rapid institution of treatment can alter an evolving event and make it impossible to determine, after the fact, whether it in fact meets the definition of anaphylaxis. This issue can be less problematic for other AEFI such as thrombocytopenia. The second issue relates to causality. Not all reports received represent causally associated adverse events because temporal association does not prove casual association. For example, background disease occurrence in the population may have caused an event to occur but still may be reported as an AEFI. This may be evident with such AEFI as GBS and thrombocytopenia. Both of these issues may lead to excess reporting and be the cause of higher than expected sensitivity and maybe much more pronounced in rare AEFI. In past studies of vaccine adverse event reporting systems, some of the reports were eliminated based on timing of the adverse events in correlation with vaccination. In this study no reports were eliminated because of the belief that that inclusion of those reports is helpful in assessing the extent of reporting.

\subsection{Implications}

This study is the first of its kind for the Canadian system. Further research and utilization of the data are necessary. Despite the inherent limitations of spontaneous systems, it is important to strive for a highly standardized system with regular measurement of characteristics like sensitivity to understand to what degree variation exists. A continuous and annual evaluation of the quality of the system is important for the purpose of improvement and may help strengthen public confidence. Spontaneous systems are not rigid in nature but rather are fluid entities, so annual assessment would be essential to any successful system. Current guidelines recommend annual assessment of sensitivity and other characteristics in order to make proper adjustments to the system that may result in increased and higher quality reporting (German 2001). Sensitivity as an assessment tool of the system holds special importance since the model to create it takes into account changes in population, coverage, and administration. This value is more useful in assessing the system than simply looking at the number of reports annually. VAERS currently conducts this type of assessment annually as a retrospective assessment of the system. 
The systems are dependent on outside reporters, so perception of those outside the system is relevant (Wysowski \& Swartz 2005). A better understanding of the system will not only raise further research questions but also will serve as a tool for hypothesis generation and quality improvement initiatives. To improve public confidence publication of these results and other results utilizing the reports must be published. This will improve public confidence for a few reasons. 1) The results will make evident to the public that their reports are being utilized. 2) The publication of these results will increase public confidence that the system receives a substantial number of reports, even for rare adverse events, at a level that is high enough to maintain the systems primary goal. 3) The system exhibits ability for response to increased reports. 4) Further efforts to improve the system are continually being made. 5) Future research will continue to better understand reporting and possibly look at greater applications and assessment of the reports.

The results show that although the overall sensitivity for each AEFI was quite variable, the system had sensitivities that were as high as seen in past research. Year by year analysis showed steady rates in more common adverse events and greater variation in more rare events. This variation over time gives greater evidence for the need of implementation of standardized case-definitions to be used in reporting. Further education of those administering vaccines coupled with incorporation of the case definitions into the reporting forms may prove to be beneficial in assisting to stabilize the fluctuation of sensitivity. This was especially apparent in the reporting of anaphylaxis, which is commonly misdiagnosed and misreported. Implementation of such casedefinitions may prove to improve specificity and PPV, and help limit variation of sensitivity overtime.

Implementation of case-definitions has been at the forefront of current international vaccine safety initiatives. The Brighton Collaboration is an international collaboration aiming to help evaluate issues related to vaccine safety. One of the major goals of the collaboration was to standardize case definitions that can be used internationally. Standardization of these case definitions would help comparability of research and help in monitoring reporting (Ball et al. 2002; Bonhoeffer et al. 2002; Kohl et al. 2007). The case-definitions as well as published studies utilizing the case-definitions are accessible online to the public. However, integration of these case definitions into the reporting forms is only now beginning.

There are published papers discussing the Brighton collaboration. The majority of the published papers has been on the discussion of the creation and need for case definitions. The case-definitions may help in data comparability in clinical trials, surveillance systems, and retrospective epidemiological studies (Clark \& Camargo 2007). It is important to note that the case-definitions are not meant to be used for the identification of a causal assessment or establishing possible management for the adverse event. Rather, they are meant to help define various levels of diagnostic certainty based on the current literature using specified criteria unique to the adverse event. The case definitions are placed at three different levels of evidence with different diagnostic criteria being 
placed in one of the three levels. These levels are used to then assess the likelihood of a causal association.

Research is also ongoing on the use and applications of these case-definitions. One published paper looked at assessing the applicability, reliability, sensitivity and specificity of applying the case definitions to the VAERS data (Kohl et al. 2008). The study used the case definitions for fever, generalized convulsive seizures, HHE, intussusceptions, nodule at injection site, and persistent crying. The study concluded that the use of the case definitions had high sensitivity and specificity when compared to clinician review of the case reports. The study confirms the importance of these casedefinitions and the need for continued development and acceptance in vaccine safety surveillance systems globally. Currently, the CAEFISS has begun plans and various projects on the implementation and utilization of these case-definitions in relation to anaphylaxis. The findings of this study strengthen the importance of this initiative.

\subsection{Future Research}

\subsubsection{PPV Analysis}

Although this is an assessment of surveillance systems, this study, along with previous research, suggests further value and benefit in the assessment of the PPV of a system as well (German 2000). Assessment of the PPV analysis will enable the researchers to better understand the quality and the types of reports being received. In this assessment of sensitivity, it is assumed that the PPV is $100 \%$, in other words, all reports received are true reports as well as casually associated. In reality, this may not hold true and many reports may be erroneous and need to be assessed on a report by report basis. A high PPV refers to a system in which a large proportion of reports received are true positives, versus a low PPV which is evidence of a system receiving many false positive reports. A low PPV may be a sign of wasted resources and the need for the implementation of education and stricter case-definitions for reporters.

Currently, Canada is in the pilot phase of applying many of these case-definitions to its systems. Applications of these case-definitions and studies on how they affect both the sensitivity and the PPV would be of importance. As evidenced by the results in this study, especially with anaphylaxis, there may be over-reporting. The implementation of case-definitions and education concerning these adverse events may help stabilize the variation in reporting many of the adverse events.

\subsubsection{Policy and Reporter Assessment}

The impact that other factors may have on the sensitivity of the reporting system is also of interest. In our current study, we assessed the specific characteristics of the AEFI itself, such as vaccine, rate of occurrence, age of patient, and timing of adverse event. Reporting may also be impacted by factors associated with the reporters, such as 
professional affiliation. The impact of professional affiliation on reporting is tied closely with the type of policy in various provinces. In some provinces the majority of vaccination is completed by public health officials rather than nurses and physicians in practice settings. Policy impacts the mandates around reporting. The policies of some provinces make adverse event reporting mandatory, while it is voluntary in others. It is unknown whether these policy variations impact the reporting system and how they would impact the sensitivity of the system.

\subsubsection{Trending Analysis Using Moving Averages}

The results of this study showed evidence of variation in the year by year analysis. It was difficult to ascertain which factors and which years were of importance; this was especially true in rarer AEFI. The methodology of moving averages has been used in business and disease outbreak research. A moving average is a form of average which has been adjusted to allow for seasonal or cyclical components of a time series. Moving average smoothing is a smoothing technique used to make the long term trends of a time series clearer.

The application of the moving average for vaccine safety has not been reported in the literature. Small studies have attempted to apply the methodology to adverse event reporting within hospitals (Morton 2009). The application of such a methodology would allow for assessment of trending as well as a potential for signaling that maybe more apparent. The application of this methodology to adverse event reporting is still in its infancy and has not been applied to sensitivity. In application to a rare event such as GBS related to influenza vaccinations (Figure 5.1), we see that this methodology limits the variation and depicts a smoother curve.

\subsection{Conclusion}

This is the first assessment of the sensitivity of the CAEFISS, and this study found that the system has reasonable ability to detect AEFI on a national level. This study found that the overall sensitivity of the CAEFISS varied from $1.0 \%$ to $136.6 \%$ for various adverse events for the years 1997-2008. CAEFISS had comparable sensitivity to previously completed studies. Many of the AEFI had sensitivity values higher than the 5\%-10\% range traditionally seen in other passive surveillance systems related to adverse events (Begaud, Martin, Haramburu, \& Moore 2002). The greatest variation of sensitivity was seen between vaccines. Rarity and timing of the AEFI also appeared to have an impact on the sensitivity. Variation of sensitivity and the variation found in the sensitivity analysis lend credence to the further development and implementations of case definitions for rarer adverse events, especially anaphylaxis. This type of analysis should be conducted annually for continued assessment of the system. Further research of other factors that impact reporting is necessary. 


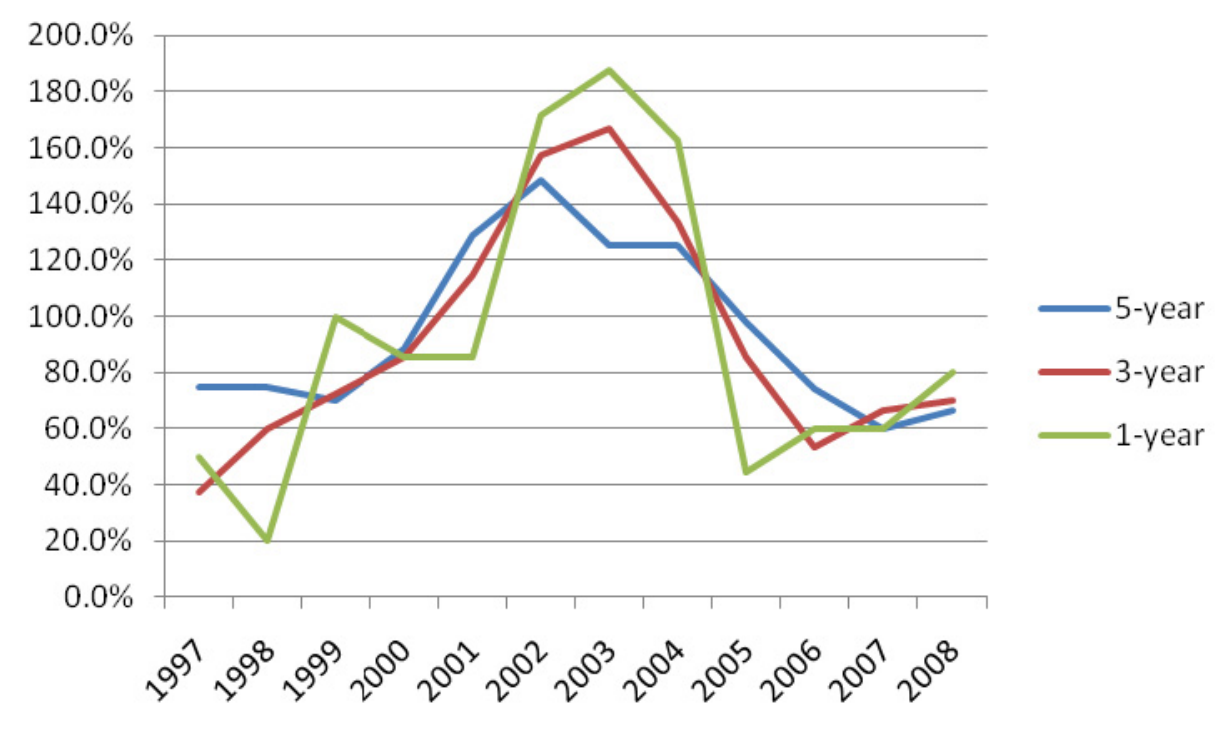

Figure 5.1 Application of Moving Averages to GBS Related to Influenza Vaccination; 1-, 3-, and 5- Year Moving Averages 


\section{LIST OF REFERENCES}

Babl, F. E., Lewena, S., \& Brown, L. (2006). Vaccination-related adverse events. Pediatr Emerg Care, 22(7), 514-519; quiz 520-512.

Ball, R., Halsey, N., Braun, M. M., Moulton, L. H., Gale, A. D., Rammohan, K., et al. (2002). Development of case definitions for acute encephalopathy, encephalitis, and multiple sclerosis reports to the vaccine: Adverse Event Reporting System. $J$ Clin Epidemiol, 55(8), 819-824.

Begaud, B., Martin, K., Haramburu, F., \& Moore, N. (2002). Rates of spontaneous reporting of adverse drug reactions in France. JAMA, 288(13), 1588.

Beland, Y. (2002). Canadian community health survey--methodological overview. Health Rep, 13(3), 9-14.

Betancourt, J. A., Hakre, S., Polyak, C. S., \& Pavlin, J. A. (2007). Evaluation of ICD-9 codes for syndromic surveillance in the electronic surveillance system for the early notification of community-based epidemics. Mil Med, 172(4), 346-352.

Bohlke, K., Davis, R. L., DeStefano, F., Marcy, S. M., Braun, M. M., \& Thompson, R. S. (2004). Epidemiology of anaphylaxis among children and adolescents enrolled in a health maintenance organization. $J$ Allergy Clin Immunol, 113(3), 536-542.

Bohlke, K., Davis, R. L., Marcy, S. M., Braun, M. M., DeStefano, F., Black, S. B., et al. (2003). Risk of anaphylaxis after vaccination of children and adolescents. Pediatrics, 112(4), 815-820.

Bonhoeffer, J., Gold, M. S., Heijbel, H., Vermeer, P., Blumberg, D., Braun, M., et al. (2004). Hypotonic-Hyporesponsive Episode (HHE) as an adverse event following immunization: Case definition and guidelines for data collection, analysis, and presentation. Vaccine, 22(5-6), 563-568.

Bonhoeffer, J., \& Heininger, U. (2007). Adverse events following immunization:

Perception and evidence. Current Opinion in Infectious Diseases, 20(3), 237-246.

Bonhoeffer, J., Heininger, U., Kohl, K., Chen, R. T., Duclos, P., Heijbel, H., et al. (2004). Standardized case definitions of adverse events following immunization (AEFI). Vaccine, 22(5-6), 547-550.

Bonhoeffer, J., Kohl, K., Chen, R., Duclos, P., Heijbel, H., Heininger, U., et al. (2002). The Brighton Collaboration: Addressing the need for standardized case definitions of adverse events following immunization (AEFI). Vaccine, 21(3-4), 298-302. 
Braun, M. M., Terracciano, G., Salive, M. E., Blumberg, D. A., Vermeer-de Bondt, P. E., Heijbel, H., et al. (1998). Report of a US public health service workshop on hypotonic-hyporesponsive episode (HHE) after pertussis immunization. Pediatrics, 102(5), E52.

Buettcher, M., Heininger, U., Braun, M., Bonhoeffer, J., Halperin, S., Heijbel, H., et al. (2007). Hypotonic-hyporesponsive episode (HHE) as an adverse event following immunization in early childhood: Case definition and guidelines for data collection, analysis, and presentation. Vaccine, 25(31), 5875-5881.

Canadian immunization guide: Final report (2005). Ottawa: Public Health Agency of Canada.

CANSIM : summary directory (2001). Ottawa: Statistics Canada.

Chen, R. T., DeStefano, F., Davis, R. L., Jackson, L. A., Thompson, R. S., Mullooly, J. P., et al. (2000). The Vaccine Safety Datalink: Immunization research in health maintenance organizations in the USA. Bull World Health Organ, 78(2), 186-194.

Chen, R. T., Mootrey, G., \& DeStefano, F. (2000). Safety of routine childhood vaccinations. An epidemiological review. Paediatr Drugs, 2(4), 273-290.

Clark, S., \& Camargo, Jr, C. A. (2007). Epidemiology of anaphylaxis. Immunology and Allergy Clinics of North America, 27(2), 145-163.

Coop, C. A., Balanon, S. K., White, K. M., Whisman, B. A., \& Rathkopf, M. M. (2008). Anaphylaxis from the influenza virus vaccine. Int Arch Allergy Immunol, 146(1), $85-88$.

DeStefano, F., \& Chen, R. T. (2000). Autism and measles, mumps, and rubella vaccine: No epidemiological evidence for a causal association. $J$ Pediatr, 136(1), 125-126.

Duclos, P., Hockin, J., Pless, R., \& Lawlor, B. (1997). Reporting vaccine-associated adverse events. Can Fam Physician, 43, 1551-1556, 1559-1560.

DuVernoy, T. S., \& Braun, M. M. (2000). Hypotonic-hyporesponsive episodes reported to the Vaccine Adverse Event Reporting System (VAERS), 1996-1998.

Pediatrics, 106(4), E52.

Ellenberg, S. S., \& Braun, M. M. (2002). Monitoring the safety of vaccines: Assessing the risks. Drug Saf, 25(3), 145-152.

Erlewyn-Lajeunesse, M., Bonhoeffer, J., Ruggeberg, J. U., \& Heath, P. T. (2007). Anaphylaxis as an adverse event following immunisation. Journal of Clinical Pathology, 60(7), 737-739. 
Fiore, A. E., Shay, D. K., Broder, K., Iskander, J. K., Uyeki, T. M., Mootrey, G., et al. (2009). Prevention and control of seasonal influenza with vaccines:

Recommendations of the Advisory Committee on Immunization Practices (ACIP), 2009. MMWR Recomm Rep, 58(RR-8), 1-52.

Fombonne, E., Zakarian, R., Bennett, A., Meng, L., McLean-Heywood, D., Fombonne, E., et al. (2006). Pervasive developmental disorders in Montreal, Quebec, Canada: Prevalence and links with immunizations. [Multicenter Study Research Support, Non-U.S. Gov't]. Pediatrics, 118(1), e139-150.

Fowler, G. L., Weintraub, E., Kennedy, A., Luman, E. T., Shui, I., Khromava, A., et al. (2008). Vaccine safety perceptions and experience with adverse events following immunization in Uzbekistan. Public Health, 122(4), 412-416.

German, R. R. (2000). Sensitivity and predictive value positive measurements for public health surveillance systems. Epidemiology, 11(6), 720-727.

German, R. R., Lee, L. M., Horan, J. M., Milstein, R. L., Pertowski, C. A., \& Waller, M. N. (2001). Updated guidelines for evaluating public health surveillance systems: Recommendations from the Guidelines Working Group. MMWR Recomm Rep, 50(RR-13), 1-35; quiz CE31-37.

Goodman, M. J., \& Nordin, J. (2006). Vaccine adverse event reporting system reporting source: A possible source of bias in longitudinal studies. Pediatrics, 117(2), 387390.

Grabenstein, J. D., \& Grabenstein, L. A. (1997). Pocket immunoFacts : Vaccines \& immunologics (pp. v.). United States: Facts and Comparisons.

Handler, S. M., Hanlon, J. T., Perera, S., Saul, M. I., Fridsma, D. B., Visweswaran, S., et al. (2008). Assessing the performance characteristics of signals used by a clinical event monitor to detect adverse drug reactions in the nursing home. AMIA Annu Symp Proc, 278-282.

Hwang, S. H., Lee, S., Koo, H. K., \& Kim, Y. (2008). Evaluation of a computer-based adverse-drug-event monitor. Am J Health Syst Pharm, 65(23), 2265-2272.

Immunization information systems progress--United States, 2006 (2008). MMWR Morb Mortal Wkly Rep, 57(11), 289-291.

Iskander, J., Pool, V., Zhou, W., \& English-Bullard, R. (2006). Data mining in the US using the Vaccine Adverse Event Reporting System. Drug Saf, 29(5), 375-384.

Jacobson, R. M. (2003). Vaccine safety. Immunology and Allergy Clinics of North America, 23(4), 589-603. 
Jadavji, T., Scheifele, D., \& Halperin, S. (2003). Thrombocytopenia after immunization of Canadian children, 1992 to 2001. Pediatr Infect Dis J, 22(2), 119-122.

Jhung, M. A., Budnitz, D. S., Mendelsohn, A. B., Weidenbach, K. N., Nelson, T. D., \& Pollock, D. A. (2007). Evaluation and overview of the National Electronic Injury Surveillance System-Cooperative Adverse Drug Event Surveillance Project (NEISS-CADES). Med Care, 45(10 Supl 2), S96-102.

Juurlink, D. N., Stukel, T. A., Kwong, J., Kopp, A., McGeer, A., Upshur, R. E., et al. (2006). Guillain-Barre syndrome after influenza vaccination in adults: A population-based study. Arch Intern Med, 166(20), 2217-2221.

Kagy, L., \& Blaiss, M. S. (1998). Anaphylaxis in children. Pediatric Annals, 27(11), 727734.

Kimmel, S. R., Burns, I. T., \& Zimmerman, R. K. (2003). Addressing immunization barriers, benefits, and risks. Journal of Family Practice, 52(1 SUPPL.), S47-S55.

Koh, Y., \& Li, S. C. (2005). A new algorithm to identify the causality of adverse drug reactions. Drug Saf, 28(12), 1159-1161.

Kohl, K. S., Gidudu, J., Bonhoeffer, J., Braun, M. M., Buettcher, M., Chen, R. T., et al. (2007). The development of standardized case definitions and guidelines for adverse events following immunization. Vaccine, 25(31), 5671-5674.

Kohl, K. S., Magnus, M., Ball, R., Halsey, N., Shadomy, S., \& Farley, T. A. (2008). Applicability, reliability, sensitivity, and specificity of six Brighton Collaboration standardized case definitions for adverse events following immunization. Vaccine, 26(50), 6349-6360.

Lasky, T., Terracciano, G. J., Magder, L., Koski, C. L., Ballesteros, M., Nash, D., et al. (1998). The Guillain-Barre syndrome and the 1992-1993 and 1993-1994 influenza vaccines. $N$ Engl J Med, 339(25), 1797-1802.

Le Saux, N., Barrowman, N. J., Moore, D. L., Whiting, S., Scheifele, D., \& Halperin, S. (2003). Decrease in hospital admissions for febrile seizures and reports of hypotonic-hyporesponsive episodes presenting to hospital emergency departments since switching to acellular pertussis vaccine in Canada: A report from IMPACT. Pediatrics, 112(5), e348.

Letourneau, M., Wells, G., Walop, W., \& Duclos, P. (2008). Improving global monitoring of vaccine safety: A quantitative analysis of adverse event reports in the WHO Adverse Reactions Database. Vaccine, 26(9), 1185-1194.

Lopez-Gonzalez, E., Herdeiro, M. T., \& Figueiras, A. (2009). Determinants of underreporting of adverse drug reactions: a systematic review. Drug Saf, 32(1), 19-31. 
Majdzadeh, R., \& Pourmalek, F. (2008). A conditional probability approach to surveillance system sensitivity assessment. Public Health, 122(1), 53-60.

Mansoor, O. (1999). Immunization safety surveillance : guidelines for managers of immunization programmes on reporting and investigating adverse events following immunization. Manila: World Health Organization, Regional Office for the Western Pacific.

Martin, B. L., Nelson, M. R., Hershey, J. N., \& Engler, R. J. (2003). Adverse reactions to vaccines. Clin Rev Allergy Immunol, 24(3), 263-276.

McAdams, M., Staffa, J., \& Dal Pan, G. (2008). Estimating the extent of reporting to FDA: a case study of statin-associated rhabdomyolysis. Pharmacoepidemiol Drug Saf, 17(3), 229-239.

McNeil, M. M., Ma, G., Aranas, A., Payne, D. C., \& Rose, C. E., Jr, (2007). A comparative assessment of immunization records in the Defense Medical Surveillance System and the Vaccine Adverse Event Reporting System. Vaccine, 25(17), 3428-3436.

Miller, E., Waight, P., Farrington, C. P., Andrews, N., Stowe, J., \& Taylor, B. (2001). Idiopathic thrombocytopenic purpura and MMR vaccine. Arch Dis Child, 84(3), 227-229.

Nebeker, J. R., Yarnold, P. R., Soltysik, R. C., Sauer, B. C., Sims, S. A., Samore, M. H., et al. (2007). Developing indicators of inpatient adverse drug events through nonlinear analysis using administrative data. Med Care, 45(10 Supl 2), S81-88.

Niu, M. T., Erwin, D. E., \& Braun, M. M. (2001). Data mining in the US Vaccine Adverse Event Reporting System (VAERS): Early detection of intussusception and other events after rotavirus vaccination. Vaccine, 19(32), 4627-4634.

Nokleby, H. (2006). Vaccination and anaphylaxis. Curr Allergy Asthma Rep, 6(1), 9-13.

O'Hagan, D. T., \& Rappuoli, R. (2004). The safety of vaccines. Drug Discov Today, 9(19), 846-854.

Pool, V., Braun, M. M., Kelso, J. M., Mootrey, G., Chen, R. T., Yunginger, J. W., et al. (2002). Prevalence of anti-gelatin IgE antibodies in people with anaphylaxis after measles-mumps rubella vaccine in the United States. Pediatrics, 110(6), e71.

Rosenthal, S., \& Chen, R. (1995). The reporting sensitivities of two passive surveillance systems for vaccine adverse events. Am J Public Health, 85(12), 1706-1709. 
Scheifele, D. W., \& Halperin, S. A. (2003). Immunization Monitoring Program, Active: A model of active surveillance of vaccine safety. Semin Pediatr Infect Dis, 14(3), 213-219.

Souayah, N., Nasar, A., Suri, M. F., \& Qureshi, A. I. (2007). Guillain-Barre syndrome after vaccination in United States a report from the CDC/FDA Vaccine Adverse Event Reporting System. Vaccine, 25(29), 5253-5255.

Stowe, J., Kafatos, G., Andrews, N., \& Miller, E. (2008). Idiopathic thrombocytopenic purpura and the second dose of MMR. Arch Dis Child, 93(2), 182-183.

Stratton, K. R., Howe, C. J., Johnston, Jr, R. B., \& Majewski, D. (1994). Adverse events associated with childhood vaccines other than pertussis and rubella: Summary of a report from the Institute of Medicine. Journal of the American Medical Association, 271(20), 1602-1605.

Stratton, K. R., Institute of Medicine (U.S.). Board on Health Promotion and Disease Prevention., \& Institute of Medicine (U.S.). Immunization Safety Review Committee. (2001). Immunization safety review : measles-mumps-rubella vaccine and autism. Washington, D.C.: National Academy Press.

Varricchio, F., Iskander, J., Destefano, F., Ball, R., Pless, R., Braun, M. M., et al. (2004). Understanding vaccine safety information from the Vaccine Adverse Event Reporting System. Pediatr Infect Dis J, 23(4), 287-294.

Verstraeten, T., Baughman, A. L., Cadwell, B., Zanardi, L., Haber, P., \& Chen, R. T. (2001). Enhancing vaccine safety surveillance: A capture-recapture analysis of intussusception after rotavirus vaccination. Am J Epidemiol, 154(11), 1006-1012.

Visweswaran, S., Hanbury, P., Saul, M., \& Cooper, G. F. (2003). Detecting adverse drug events in discharge summaries using variations on the simple Bayes model. AMIA Annu Symp Proc, 689-693.

Woo, E. J., Ball, R., Burwen, D. R., \& Braun, M. M. (2008). Effects of stratification on data mining in the US Vaccine Adverse Event Reporting System (VAERS). Drug Safety, 31(8), 667-674.

Wysowski, D. K., \& Swartz, L. (2005). Adverse drug event surveillance and drug withdrawals in the United States, 1969-2002: The importance of reporting suspected reactions. Arch Intern Med, 165(12), 1363-1369.

Zanardi, L. R., Haber, P., Mootrey, G. T., Niu, M. T., \& Wharton, M. (2001). Intussusception among recipients of rotavirus vaccine: Reports to the vaccine adverse event reporting system. Pediatrics, 107(6), E97. 
Zent, O., Arras-Reiter, C., Broeker, M., \& Hennig, R. (2002). Immediate allergic reactions after vaccinations--a post-marketing surveillance review. Eur J Pediatr, 161(1), 21-25.

Zhou, W., Pool, V., Iskander, J. K., English-Bullard, R., Ball, R., Wise, R. P., et al. (2003). Surveillance for safety after immunization: Vaccine Adverse Event Reporting System (VAERS)--United States, 1991-2001. MMWR Surveill Summ, 52(1), 1-24. 


\section{VITA}

Mina Tadrous was born in Alexandria, Egypt on October 23, 1983. He is the Son of Ted and Madeleine Tadrous, brother to Mary and Mark Tadrous. They immigrated to Toronto, Canada in 1991. He graduated from Newmarket High School and attended the University of Toronto where he completed his undergraduate course work. He received his Pharmacy training and a Doctor of Pharmacy from the Albany College of Pharmacy in Albany, NY in May 2008. After graduation he pursued a Drug Information and Health Outcomes Residency at the University of Tennessee, MedCommunications Inc., and St. Jude Children's Research Hospital. He concurrently completed his Master's in Health Outcomes and Policy research, and is planning to graduate in May 2010. 